\title{
INSTRUMENTOS LÍTICOS DE CUARZO, PRÁCTICAS SOCIALES Y VIDA CAMPESINA DURANTE EL PRIMER MILENIO DE LA ERA EN EL ESTE DE CATAMARCA, ARGENTINA
}

\author{
Débora Egea* y Enrique Moreno**
}

Fecha de recepción: 29 de diciembre de 2020

Fecha de aceptación: 25 de mayo de 2021

\section{RESUMEN}

En la reproducción social cotidiana, las poblaciones humanas utilizan diferentes herramientas para resolver actividades diarias. Una de las formas de acceder a estos instrumentos es a través del estudio de la formatización de filos, puntas y superficies líticas. En este trabajo nos interesa introducirnos en la forma en que manufacturaron sus herramientas las poblaciones que habitaron la Sierra de El Alto-Ancasti (Catamarca, Argentina) durante el primer milenio de la era. Para ello, se evalúan las características técnicas, morfológicas y funcionales de los instrumentos líticos recuperados en diferentes sitios excavados en el este catamarqueño, para luego analizar las diferencias presentes en los distintos sitios arqueológicos estudiados. Además, este trabajo constituye un aporte al análisis de los instrumentos manufacturados en cuarzo, materia prima que presenta algunas particularidades en cuanto a su dureza y calidad para la talla, pero que representa prácticamente la totalidad de la tecnología lítica en sitios trabajados en una vasta zona del centro-norte de la Argentina.

Palabras clave: tecnología lítica-cuarzo-Sierra de El Alto-Ancasti-campesinos-primer milenio d.C.

\footnotetext{
* Centro de Investigaciones y Transferencia de Catamarca (CITCA - CONICET/UNCA). E-mail: deb.egea@ gmail.com

** Centro de Investigaciones y Transferencia de Catamarca (CITCA - CONICET/UNCA), Escuela de Arqueología (UNCA). E-mail: enalmor@gmail.com
} 
Relaciones de la Sociedad Argentina de Antropología 46 (1), enero-junio 2021: 145-176

QUARTZ LITHIC INSTRUMENTS, SOCIAL PRACTICES AND PEASANT LIFE DURING THE FIRST MILLENIUM OF THE ERA AT EASTERN CATAMARCA, ARGENTINA

\section{ABSTRACT}

Human populations used different lithic tools in order to address daily activities in everyday social reproduction. One way of approaching these instruments is by studying how lithic edges, points and surfaces were formatted. In this work, we are interested in introducing tool manufacturing by human populations that inhabited El Alto-Ancasti mountains (Catamarca, Argentina) during the first millennium of the era. For this purpose, we evaluate technical, morphological and functional characteristics of lithic instruments retrieved from excavations in different sites of eastern Catamarca, and then analyze the differences among the latter. In addition, this work is a contribution to the study of instruments made of quartz, a raw material that displays particular features in terms of hardness and quality for knapping. It also practically represents the whole lithic technology found in archaeological sites in a wide area of central and northern Argentina.

Keywords: lithic technology - quartz - El Alto-Ancasti mountains - peasents - first millennia a.C.

\section{INTRODUCCIÓN}

Las poblaciones humanas que habitaron el este catamarqueño durante la segunda mitad del primer milenio de la era establecieron una fuerte relación con el cuarzo para la manufactura de diferentes tipos de instrumentos utilizados en la reproducción de las actividades cotidianas (Moreno y Sentinelli 2014; Moreno 2015; Egea 2016, 2018; Egea y Gerola 2020; Moreno y Egea 2020). Estos participaron de múltiples tareas como cortar, raspar, punzar, ahuecar, cincelar, etc.

Debemos considerar que este mineral se caracteriza por poseer una muy alta dureza y gran dificultad para controlar la fractura al ejecutar la reducción de los núcleos para obtener formas base, sobre todo por la presencia de inclusiones o planos de fractura. A su vez, resulta difícil identificar rasgos diagnósticos en estos materiales, siguiendo las propuestas generales del análisis tecno-morfológico y morfológico funcional que utilizamos para realizar los estudios específicos (Aschero 1975, 1983). Sin embargo, se han podido alcanzar avances relevantes en la comprensión de las elecciones de los talladores y las talladoras y en los criterios de registro de las técnicas aplicadas por estas poblaciones sobre esta materia prima que no se comporta de la misma manera que otras, como el basalto, la obsidiana o la calcedonia, de mejor calidad para la talla (Mourre 1996; Fábregas Valcarce y Rodríguez Rellán 2008; De la Peña 2011; Driscoll 2011; Pautassi y Sario 2014; Pargeter y De la Peña 2017; Sánchez Yustos et al. 2017; Egea 2018; Pautassi 2018).

Así, el análisis de la tecnología lítica recuperada durante los trabajos de campo realizados en la Sierra de El Alto-Ancasti, Catamarca, nos ha permitido un acercamiento a la forma en que las personas obtuvieron los nódulos, los redujeron para obtener formas base y manufacturaron los filos (Moreno y Sentinelli 2014; Moreno 2015; Egea 2016, 2018; Moreno y Egea 2016, 2020). En esos trabajos, sumados a los de autores en otros sitios arqueológicos del centro-norte argentino, resalta el cuarzo como la materia prima utilizada casi exclusivamente para la manufactura de estos instrumentos (Pautassi y Sario 2014; Sario y Pautassi 2015; Caminoa 2016; Vilches 2016; Carrera Aizpitarte 2017; Reinoso 2017; Brizuela 2018; Gerola 2018; Montegú 2018; Pautassi 2018; Sario y Salvatore 2018; Borgo et al. 2019). Allí, se ponen de manifiesto las diversas estrategias tecnológicas utilizadas por las poblaciones humanas para enfrentar las dificultades propias del cuarzo para la reducción y la participación de estos instrumentos en la reproducción social.

Nos interesa concentrarnos aquí en un aspecto específico de la tecnología lítica tallada que tiene que ver con los instrumentos manufacturados, aquellos que fueron utilizados, o por 
lo menos preparados, para cumplir actividades concretas y que participaron tanto de prácticas diarias y simples, como de algunas más esporádicas y específicas. Sin embargo, no nos interesa solamente una muy necesaria caracterización tecnológica de estos instrumentos (que nos permite acercarnos a su historia de manufactura o a su posible funcionalidad, buscando caracterizar las decisiones tecnológicas recurrentes o particulares), sino que también creemos imprescindible la interacción interpretativa con los contextos de los cuales provienen estos materiales. Es decir, para la interpretación de estos conjuntos es central comprender y evaluar el lugar en el cual fueron encontrados y su asociación con las actividades allí realizadas y con otras materialidades, ya que esto nos aporta indicios de las prácticas ejecutadas.

En este sentido, se compara la tecnología lítica de cuatro sitios arqueológicos con características diferentes en relación con su arquitectura, ubicación espacial y modalidad de ocupación, pero sincrónicos. Corresponden a dos compuestos domésticos, una cueva con arte rupestre y un conjunto de campamentos transitorios. Uno de los compuestos domésticos se encuentra ubicado en el pastizal de altura mientras que el otro, así como la cueva con pinturas y los campamentos transitorios se localizan en el bosque seco estacional. Consideramos entonces que, además de la información que nos pueden brindar los contextos para comprender los conjuntos líticos tallados, estos pueden aportar resultados relevantes para fortalecer las interpretaciones sobre cada uno de estos espacios.

Por ello, el objetivo general del trabajo es doble: por un lado aportar al conocimiento de la tecnología lítica tallada en cuarzo, particularmente a la forma de interpretar los instrumentos manufacturados en la Sierra de El Alto-Ancasti; y por el otro, contribuir a la caracterización de las poblaciones que manufacturaron y usaron estos instrumentos, contribuyendo a determinar las prácticas sociales en las que participaron durante la segunda mitad del primer milenio de la era en la Sierra de El Alto-Ancasti.

Los objetivos específicos están vinculados a caracterizar los instrumentos registrados en distintos sitios investigados del área de estudio, centrándonos, por un lado, en identificar las formas base utilizadas, fragmentación, tamaños y otras variables generales, y por el otro, aspectos específicos de los filos, vinculados a su forma, ángulo, técnica de manufactura y posible funcionalidad. Además, en términos metodológicos proponemos la simplificación de ciertos grupos tipológicos en algunas categorías que abarquen actividades funcionales similares. Esto es a los fines de priorizar la identificación de rasgos generales que nos acerquen a las potenciales funciones de los instrumentos, antes de centrarnos en detalles tecnológicos, muchas veces imposibles de registrar en cuarzo. Luego, la integración de la caracterización de los instrumentos será cruzada con la información contextual para evaluar las interpretaciones funcionales de cada sitio y comparar los espacios investigados.

Esto nos brindará información sobre las elecciones tecnológicas vinculadas al cuarzo como materia prima, así como también sobre las diferencias y semejanzas que pueden existir entre los diferentes contextos trabajados. Partimos entonces de la idea de que dadas las diferentes funcionalidades de los sitios arqueológicos y las distintas ubicaciones ambientales, los conjuntos instrumentales deberían corresponderse con estas diferencias. Pero además, en el caso de cumplirse estas diferencias, nos interesa reflexionar acerca del porqué de estas, así como también bucear en comprender el origen de las similitudes presentes.

\section{TECNOLOGÍA LÍTICA TALLADA EN CUARZO}

Tal como se planteó antes, la tecnología lítica tallada en cuarzo presenta algunas particularidades, tanto al momento de realizar la reducción y manufactura, como al efectuar el análisis de estos conjuntos materiales. Esta situación ha sido planteada por investigadores e investigadoras 
a escala global y regional, generándose intensos debates y aportes a partir de los que se propuso salir del flint syndrome (Knutsson 1998) que implica la necesidad de entender las dinámicas de fractura particulares del cuarzo de manera diferente a las de otras materias primas de mejor calidad para la talla, así como para el registro de rasgos tecnológicos diagnósticos. A partir de esta situación, se generó incluso una suerte de polémica tendiente a plantear si el cuarzo debía ser analizado a partir de una metodología particular, específica para esta materia prima (de Lombera Hermida 2009; Driscoll 2009), o si era necesaria la utilización de métodos de análisis efectivos para otras materias primas (Ballin 2004; Beardsell 2013). Pero aquí no nos convoca discutir sobre esta temática, sino más bien hacer referencia a esta característica del cuarzo como materia prima y la relevancia que pueden tener aportes como el de este trabajo.

Son muchas las investigaciones que se han centrado en este punto, vinculadas a diversas preguntas, como el comportamiento del cuarzo frente a la talla, el uso de la técnica bipolar, las características de los desechos indiferenciados, entre otras preguntas (Callahan 1987; Bisson 1990; Ballin 2004, 2008; Orton 2008; de Lombera Hermida 2009; Diez Martín et al. 2009; Driscoll 2010; Tallavaara et al. 2010; De La Peña y Wadley 2014; Pautassi y Sario 2014; Byrne et al. 2016; Manninen 2016; Sánchez Yustos et al. 2017; Pautassi 2018). Algunos de estos trabajos se han centrado en responder preguntas concretas acerca de la preparación de ciertos tipos de instrumentos o técnicas específicas como la miniaturización o la interpretación funcional y tecnológica alrededor de las pieces equilles (Diez Martín et al. 2009; De la Peña y Wadley 2014; Pargeter y De la Peña 2017) u otras discusiones en torno al comportamiento del cuarzo en contextos paleolíticos de poblaciones cazadoras-recolectoras y de alta movilidad. En estos casos, generalmente, el aprovechamiento del cuarzo tenía que ver con su ubicación conspicua en el paisaje, pero también con la posibilidad de obtener filos aptos para la realización de diferentes tareas ya sea a través de talla directa o de talla bipolar (Callahan 1987; Tallavara et al. 2010; Manninen 2016).

Estos aportes, además de importantes avances a nivel regional (Pautassi y Sario 2014; Sario y Pautassi 2015; Gerola 2018; Montegú 2018; Pautassi 2018; entre otros) han sido centrales para comprender algunas particularidades de la tecnología lítica en la Sierra de El Alto-Ancasti. Entre ellas, además de la altísima proporción de cuarzo como materia prima (96\%), podemos remarcar la presencia de porcentajes muy altos de desechos indiferenciados que varían entre el $13 \%$ y el $20 \%$ de la muestra total, la utilización de la talla directa y bipolar para la reducción de núcleos y el aprovechamiento de formas base simples y poco estandarizadas para la preparación de instrumentos (Moreno y Sentinelli 2014; Moreno 2015; Egea 2016, 2018; Moreno y Egea 2016, 2020).

Ahora bien, generalmente, los conjuntos de instrumentos manufacturados en cuarzo muestran poca inversión de trabajo, remarcado en el retoque unifacial sumario, la ausencia o escasa preparación de las formas base y la alta fragmentación que pueden presentar. Sin embargo, creemos que si nos centramos en estos rasgos e intentamos una propuesta que nos acerque a las actividades realizadas por estos instrumentos y su vinculación con los contextos específicos, podremos obtener mayor cantidad de información que si nos concentramos solamente en sus características tecnológicas y en la clara expeditividad y simplicidad de su manufactura. Pero antes de continuar, es necesario caracterizar los paisajes culturales de la Sierra de El Alto-Ancasti y de los sitios arqueológicos de los cuales provienen las muestras analizadas en este trabajo.

\section{LA SIERRA DE EL ALTO-ANCASTI}

La investigación que aquí se presenta se centra en sitios arqueológicos que se ubican en la Sierra de El Alto-Ancasti, localizada en el este de la provincia de Catamarca, Argentina. Esta sierra, de unos $140 \mathrm{~km}$ de longitud, divide el Valle Central de la ciudad de Catamarca de la llanura chaco-santiagueña y presenta altitudes máximas que alcanzan los 2.000 m s.n.m. Su ladera 
oriental desciende suavemente hasta la llanura chaco-santiagueña, mientras que la occidental lo hace abruptamente hacia el valle central de Catamarca (figura 1). Esta situación forma una serie de pisos altitudinales con características ambientales muy diferentes (Mórlans 1995). El primero se localiza en la ladera media y baja (entre 700 y 1500 m s.n.m.), y presenta rasgos de bosque seco estacional neotropical, le sigue un piso de arbustos y pastos (entre 1.500 y 1.800 m s.n.m.) y, finalmente, los pastizales ubicados en la cumbre (1.800-2.000 m s.n.m.).

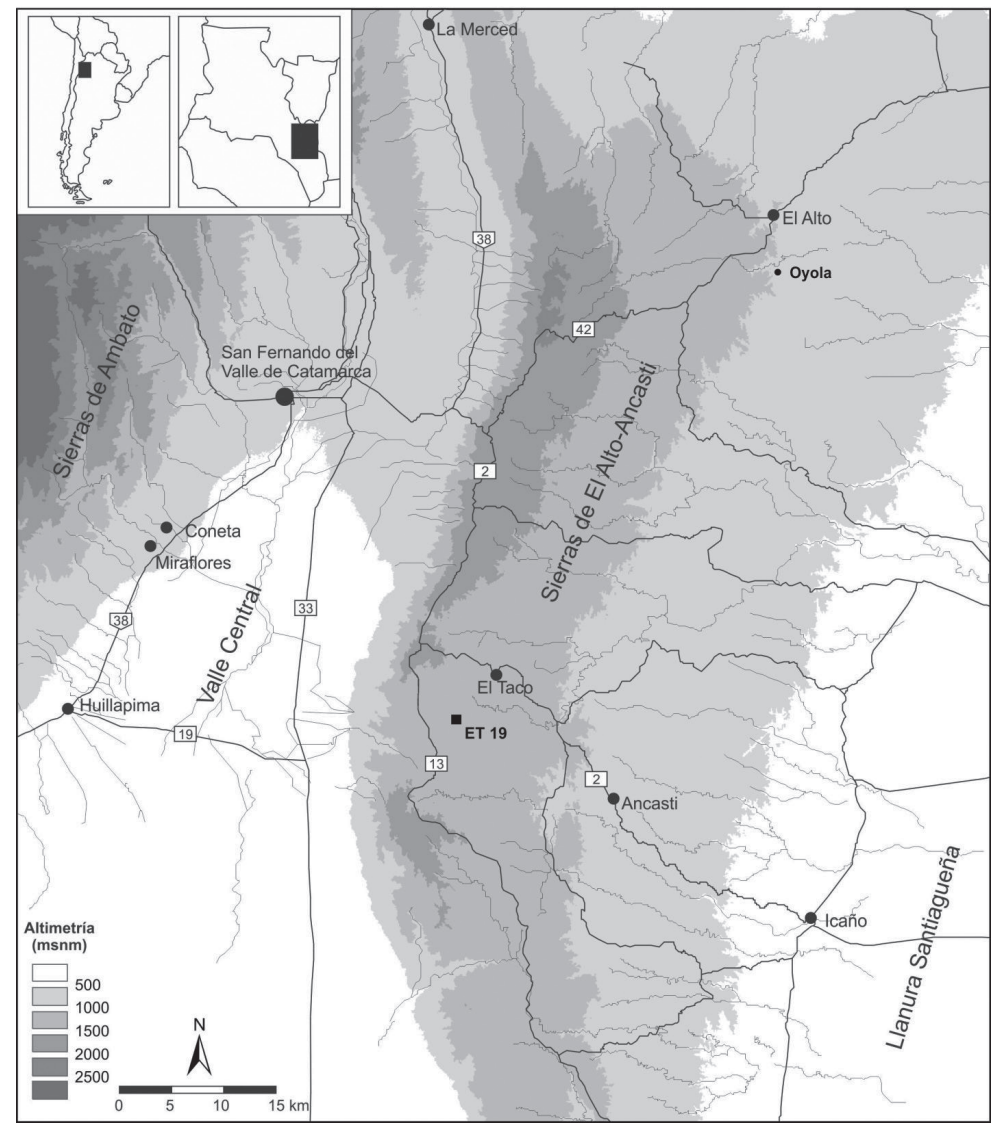

Figura 1. Mapa de ubicación de las áreas de estudio en la Sierra de El Alto-Ancasti

En los últimos quince años, en los cuales se sistematizaron las investigaciones arqueológicas en la zona, se han logrado avances importantes en torno a los contextos y paisajes culturales locales, indagando en las particularidades de las formas de vida en distintas temporalidades y pisos ecológicos en esta área (Nazar 2003; Dlugosz 2005; Quesada et al. 2012; 2016; Zuccarelli 2012, 2020; Egea 2016; Gastaldi et al. 2016; Barot 2017; Gheco 2017; Gordillo et al. 2017; Moreno y Ahumada 2018; entre otros). Con nuestro equipo de investigación realizamos estudios en numerosos sitios a lo largo de la sierra -tanto en la zona cumbral (El Taco) como en el área de bosque seco estacional (Oyola) - que muestran una forma de construcción del espacio social ligado a un importante desarrollo de la arquitectura, así como de las prácticas de producción agrícola, con ocupaciones prolongadas y permanentes (Quesada et al. 2012, 2016).

Los datos obtenidos a través de análisis espaciales, relevamientos y excavaciones estratigráficas muestran la presencia de poblaciones asentadas, con viviendas y terrazas agrícolas, correspondientes a distintos momentos del primer milenio d.C. Esto delinea paisajes campesinos 
permanentes, autosuficientes y de larga duración que establecieron fuertes relaciones con los recursos locales en una economía diversificada con prácticas agrícolas (Quesada et al. 2012, 2016; Gastaldi et al. 2016; Barot 2017; Zuccarelli 2020). Las potenciales diferencias en los distintos pisos ecológicos identificados en la Sierra de el Alto-Ancasti resultan relevantes en torno a la construcción y reproducción de los paisajes culturales en la zona.

En este trabajo, nos centraremos en dos de estos ambientes que se forman en la ladera oriental de la Sierra de El Alto-Ancasti: el pastizal de altura ubicado en la cumbre de la sierra (El Taco) y el bosque seco estacional (Oyola) (Aceñolaza et al. 1983; Mórlans 1995; Mogni et al. 2015).

En la localidad de El Taco se realizó una prospección intensiva que permitió identificar diecinueve conjuntos de estructuras arquitectónicas (ubicadas en los sectores más elevados), mientras que prácticamente en la totalidad de las laderas que descienden hacia los colectores hídricos se identificó la construcción de terrazas agrícolas. Estos rasgos permitieron caracterizar el paisaje campesino desarrollado durante la segunda mitad del primer milenio de la era cristiana (Quesada et al. 2012; Zuccarelli 2020).

Para comprender algunas particularidades sobre la escala doméstica, se realizó la excavación de dos recintos del sitio El Taco 19 (ET19) (figura 2). Este sitio exhibe muros con una significativa secuencia constructiva: la base presenta una doble hilera de lajas ubicadas de forma vertical clavadas en el sedimento, que puede alcanzar una altura de $1 \mathrm{~m}$; la secuencia se completada mediante la colocación de bloques irregulares, aunque con la selección de las caras más regulares hacia el interior del recinto. El intersticio entre las lajas fue rellenado con tierra y cascajo, mientras que se observa mortero en el caso de los bloques que completan los muros. El recinto 1 presenta un tamaño aproximado de $5 \mathrm{~m}$ de lado con una abertura hacia el sur, mientras que el recinto 2 presenta mayores dimensiones y, aparentemente, habría estado abierto hacia el sur. Fue interpretado como un posible espacio abierto, probablemente sin techar o con techado parcial. Además de la excavación de estos espacios se inició la excavación de otro, exterior, asociado a la abertura del recinto 1, denominado R3 (Quesada y Gastaldi 2013; Ahumada et al. 2019; Barot et al. 2019).

La excavación estratigráfica permitió interpretar este espacio como una unidad doméstica -que muestra modificaciones arquitectónicas y funcionales- donde se realizaron múltiples actividades, entre ellas, la cocción y servido de alimentos, el almacenamiento y la realización

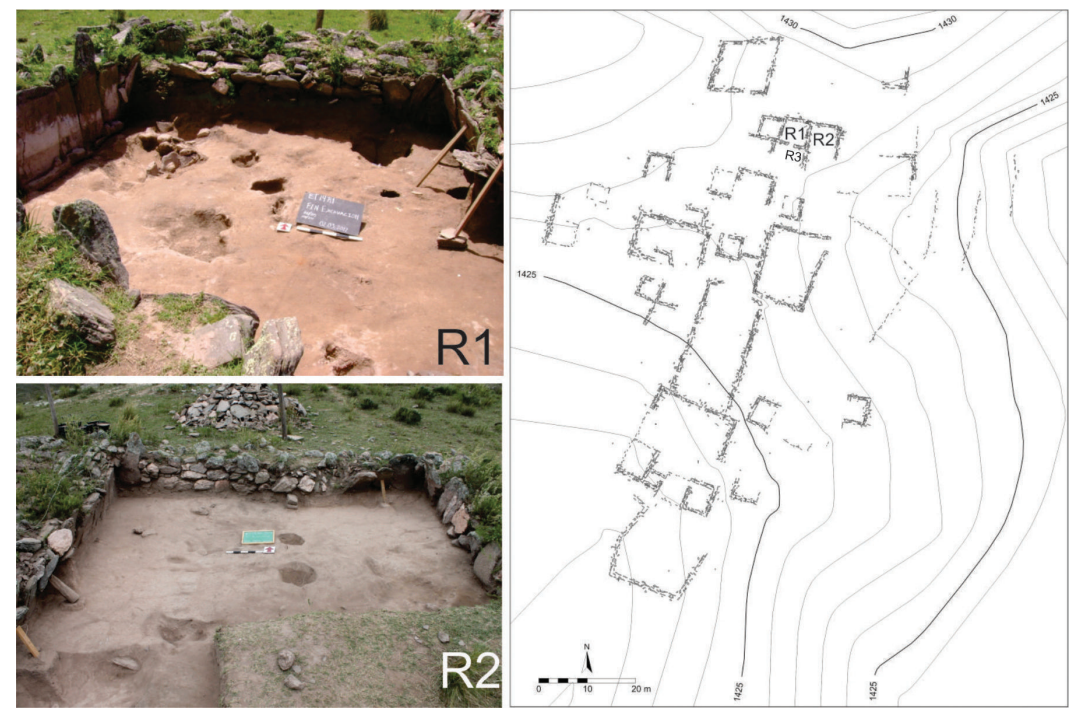

Figura 2. Derecha. Croquis de El Taco 19, donde se indican los recintos excavados. Arriba a la izquierda, detalle del recinto 1 (R1) de ET19. Abajo a la izquierda, detalle del recinto 2 (R2) de ET19 
de secuencias de producción líticas. Esto último pudo ser interpretado a partir del estudio de los distintos materiales líticos tallados recuperados, donde se observan núcleos, percutores, desechos de talla e instrumentos manufacturados (tabla 1), así como también del análisis de sus dimensiones y evidencias de utilización, tanto de talla a mano alzada como bipolar (Quesada et al. 2012; Quesada y Gastaldi 2013; Ahumada y Moreno 2015-16; Moreno 2015; Moreno y Ahumada 2018; Ahumada et al. 2019; Moreno y Egea 2020)

La otra localidad en la que desarrollamos nuestras investigaciones es Oyola, la cual se ubica en el piso altitudinal de bosque seco estacional, a unos 800 m s.n.m. (figura 1). En este espacio se han documentado e iniciado trabajos de investigación vinculados a un conjunto de al menos 38 cuevas y aleros con arte rupestre (Gheco 2012, 2017; Gastaldi et al. 2016). Una de estas cuevas es Oyola 7 (Oy7) (figura 3), ubicada en la base de una gran roca de granito, en el sector más elevado de un cerro, denominado "Cerro de Oyola".

La cueva posee dos accesos en sus extremos este y oeste. Al oeste, la entrada es de escasa altura, de no más de $1 \mathrm{~m}$, y se abre a un gran playón pétreo formado por una roca granítica desde donde pueden obtenerse amplias visuales del paisaje circundante. La boca hacia el oeste, si bien es más amplia, tiene un acceso más complejo debido a la existencia de grandes rocas y abundante vegetación. Su interior posee un tamaño aproximado de $14 \mathrm{~m}$ de largo, $4 \mathrm{~m}$ de ancho y 1,6 m de alto, lo cual la convierte en una de las cuevas más grandes de la localidad de Oyola. En su interior fueron registrados 77 motivos rupestres en colores negros, blancos y rojos (Gastaldi et al. 2016; Gheco 2017).

Esta cueva fue excavada en su totalidad, lo que permitió interpretar una secuencia de eventos de mayor intensidad en la ocupación, principalmente vinculados con el encendido de fogones y la realización de algunas actividades y momentos de menor intensidad en la ocupación, en los cuales se depositaban estratos cobertores, y en los cuales, las evidencias culturales fueron mínimas (Gastaldi et al. 2016; Gheco 2017). En relación con la tecnología lítica, se ha interpretado que las secuencias de producción se habrían realizado en otro lugar, ya que prácticamente no se han recuperado núcleos ni percutores en el interior de la cueva y, además, los desechos de talla presentan tamaños relativamente inferiores que los instrumentos, por lo que no se habrían ejecutado las secuencias de producción completas en el interior de Oyola 7 (tabla 1) (Egea 2016, 2018; Gastaldi et al. 2016; Moreno y Egea 2016; Gheco 2017; Egea y Gerola 2020).

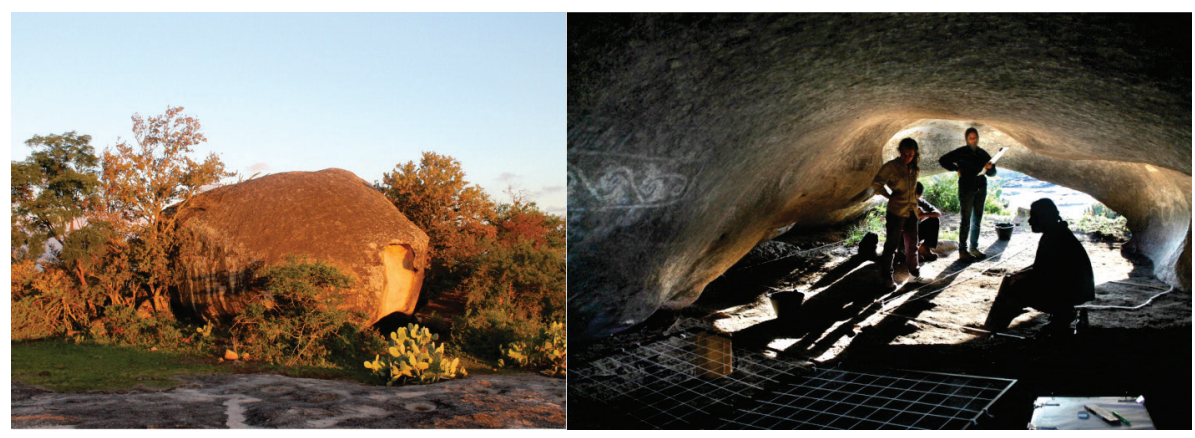

Figura 3. Vista general del exterior (izquierda) y del interior (derecha) de la cueva 7 de Oyola

Una particularidad relevante que ha mostrado el "Cerro de Oyola" es la ausencia de otras evidencias de construcciones prehispánicas (Quesada et al. 2016). Es por ello que la vida social pareció centrarse en los espacios rupestres. Solamente identificamos algunos bloques en posición vertical, en una explanada cercana a la cueva Oyola 7. Al excavar este espacio, denominado Oyola 31 (Oy31), se identificaron pequeños muros de piedra que conformaban una suerte de zócalos con 


\begin{tabular}{|c|c|c|c|c|c|c|c|c|c|c|c|}
\hline 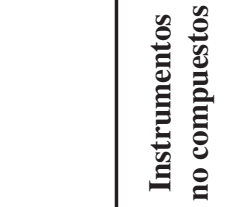 & $N$ & 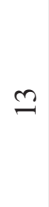 & - & & 0 & हैँ & 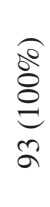 & $\begin{array}{l}\stackrel{8}{8} \\
8 \\
0 \\
= \\
0\end{array}$ & $\begin{array}{l}8 \\
8 \\
8 \\
= \\
\bar{n}\end{array}$ & \begin{tabular}{l}
0 \\
8 \\
8 \\
\hdashline \\
$a$
\end{tabular} & $\begin{array}{l}\stackrel{8}{8} \\
\stackrel{8}{8} \\
\underset{\sigma}{ \pm}\end{array}$ \\
\hline 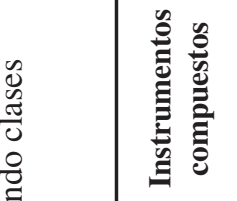 & $m$ & $r$ & - & ' & $=$ & 乙 & $\begin{array}{l}\hat{\rho} \\
\text { an } \\
a \\
a\end{array}$ & $\underset{m}{\stackrel{\sigma}{\sigma}}$ & 1 & $\begin{array}{l}Ð \varrho \\
\Xi \\
\Xi\end{array}$ & $\begin{array}{l}\widehat{d} \\
\underset{0}{0} \\
0\end{array}$ \\
\hline 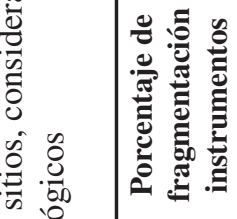 & 官 & $\stackrel{\stackrel{\circ}{+}}{\stackrel{5}{+}}$ & $\begin{array}{l}\stackrel{8}{\sigma} \\
\stackrel{b}{+}\end{array}$ & $\stackrel{\circ}{+}$ & $\stackrel{8}{8}$ & $\underset{\text { Z }}{Z_{\mathbf{I}}}$ & $\frac{d}{\frac{d}{d}}$ & ' & 1 & I & $\begin{array}{l}\widehat{d} \\
0 \\
0 \\
0 \\
\text { d }\end{array}$ \\
\hline 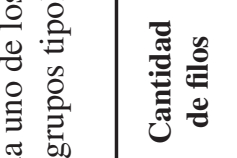 & ๙ & $\overline{0}$ & $\bar{n}$ & $a$ & $\frac{\nabla}{m}$ & 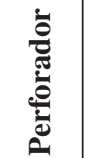 & $\stackrel{\overbrace e}{=}$ & ' & ' & ' & $\begin{array}{l}\widehat{d} \\
\hat{e} \\
\hat{e}\end{array}$ \\
\hline 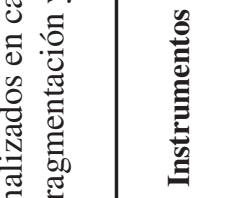 & $\begin{array}{l}0 \\
i n \\
n \\
n \\
\infty\end{array}$ & $\begin{array}{l}\stackrel{8}{\mathfrak{d}} \\
\stackrel{n}{2}\end{array}$ & $\begin{array}{l}\widehat{o} \\
\dot{m} \\
\stackrel{9}{q} \\
a\end{array}$ & $\begin{array}{l}a \\
0 \\
a \\
a\end{array}$ & 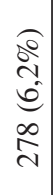 & 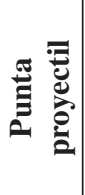 & ' & $\begin{array}{l}0 \\
60 \\
0 \\
a \\
0\end{array}$ & ' & ' & $\begin{array}{l}a \\
a \\
a \\
a\end{array}$ \\
\hline 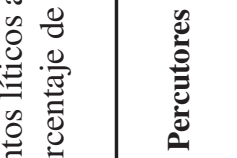 & $\begin{array}{l}\hat{0} \\
\stackrel{0}{2} \\
e \\
n\end{array}$ & ' & $\begin{array}{l}\hat{e} \\
e \\
e \\
e \\
\dot{v}\end{array}$ & ' & $\begin{array}{l}\widehat{Q} \\
\stackrel{1}{a} \\
e \\
a\end{array}$ & 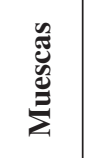 & $\begin{array}{l}\widehat{d} \\
\stackrel{a}{d} \\
\text { d }\end{array}$ & $\begin{array}{l}\frac{\partial}{\vec{c}} \\
\stackrel{\vec{c}}{=} \\
\vec{v}\end{array}$ & $\begin{array}{l}\widehat{o} \\
\infty \\
9 \\
n\end{array}$ & $\begin{array}{l}\stackrel{\varrho}{0} \\
\Xi \\
\Xi\end{array}$ & $\underset{8}{\stackrel{8}{2}}$ \\
\hline $\begin{array}{l}0 \\
\tilde{J} \\
\frac{0}{00} \\
\frac{0}{0}\end{array}$ & $\underset{\cong}{\stackrel{\overbrace \varrho}{\leftrightarrows}}$ & 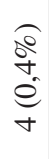 & $\begin{array}{c}\hat{o} \\
n \\
e \\
\infty\end{array}$ & 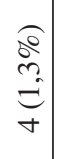 & $\begin{array}{l}\hat{e} \\
\stackrel{2}{e} \\
\dot{m}\end{array}$ & 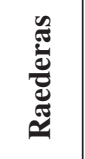 & $\begin{array}{l}\widehat{\sigma} \\
\sigma \\
\Xi \\
=\end{array}$ & $\begin{array}{l}\widehat{0} \\
\infty \\
0 \\
= \\
=\end{array}$ & $\begin{array}{l}\widehat{\sigma} \\
\sigma \\
0 \\
m\end{array}$ & $\begin{array}{l}\stackrel{\sigma}{0} \\
\Xi \\
\Xi\end{array}$ & 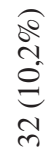 \\
\hline 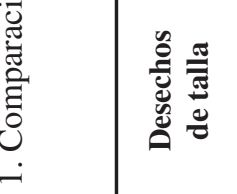 & 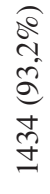 & 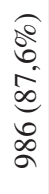 & 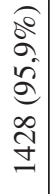 & 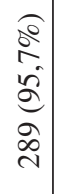 & 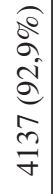 & 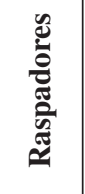 & 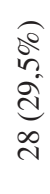 & $\begin{array}{l}\widehat{d} \\
\vec{d} \\
\vec{d} \\
\vec{m}\end{array}$ & $\begin{array}{l}\widehat{\sigma} \\
\infty \\
9 \\
0 \\
n\end{array}$ & $\begin{array}{l}\widehat{d} \\
i \\
d \\
d \\
\text { d }\end{array}$ & 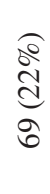 \\
\hline 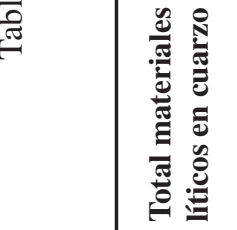 & $\stackrel{\text { }}{\bumpeq}$ & $\stackrel{2}{\Xi}$ & $\stackrel{\text { 字 }}{\stackrel{+}{+}}$ & $\stackrel{\mathcal{D}}{\mathrm{D}}$ & $\begin{array}{l}n \\
2 \\
q\end{array}$ & 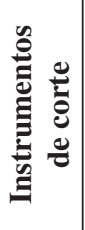 & \begin{tabular}{l}
$\widehat{\partial}$ \\
$\sigma$ \\
$\infty$ \\
\multirow{0}{\sigma}{}
\end{tabular} & $\begin{array}{l}20 \\
\stackrel{2}{2} \\
\frac{n}{2} \\
\infty\end{array}$ & \begin{tabular}{l}
$\widehat{o}$ \\
$n$ \\
\multirow{5}{*}{} \\
$\infty$ \\
$\infty$
\end{tabular} & 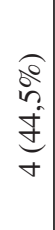 & 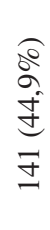 \\
\hline 汽 & $\underset{\vec{x}}{\overrightarrow{7}}$ & $\hat{b}$ & 帘 & $\overline{\tilde{d}}$ & हैّ & & $\stackrel{\theta}{\overrightarrow{7}}$ & $\hat{\sigma}$ & 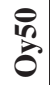 & $\overline{\tilde{b}}$ & हैं \\
\hline
\end{tabular}


función estructural que habrían contribuido a sostener empalizadas de postes que funcionaron posiblemente como cerramientos hechos con material perecedero (figura 4). Parecieran formar pequeños campamentos transitorios, utilizados por cortos períodos de tiempo, abandonados y luego vueltos a construir en otra posición. Esto lo marca la presencia de un caso de dos muros superpuestos, pero con distinta orientación y la posición no alineada de algunos agujeros de poste. Oyola 31 puede ser interpretado como una, o varias estructuras de vivienda, probablemente sucesivas y en parte superpuestas. Esta técnica constructiva poco elaborada podría vincularse a una expectativa de uso eventual (Quesada et al. 2016). En este caso, a pesar de que se analizó solo una muestra del conjunto lítico total recuperado (37\%), los resultados muestran evidencias de la realización de las distintas etapas de producción lítica, similar a lo observado en ET19 (tabla 1) (Quesada et al. 2016).
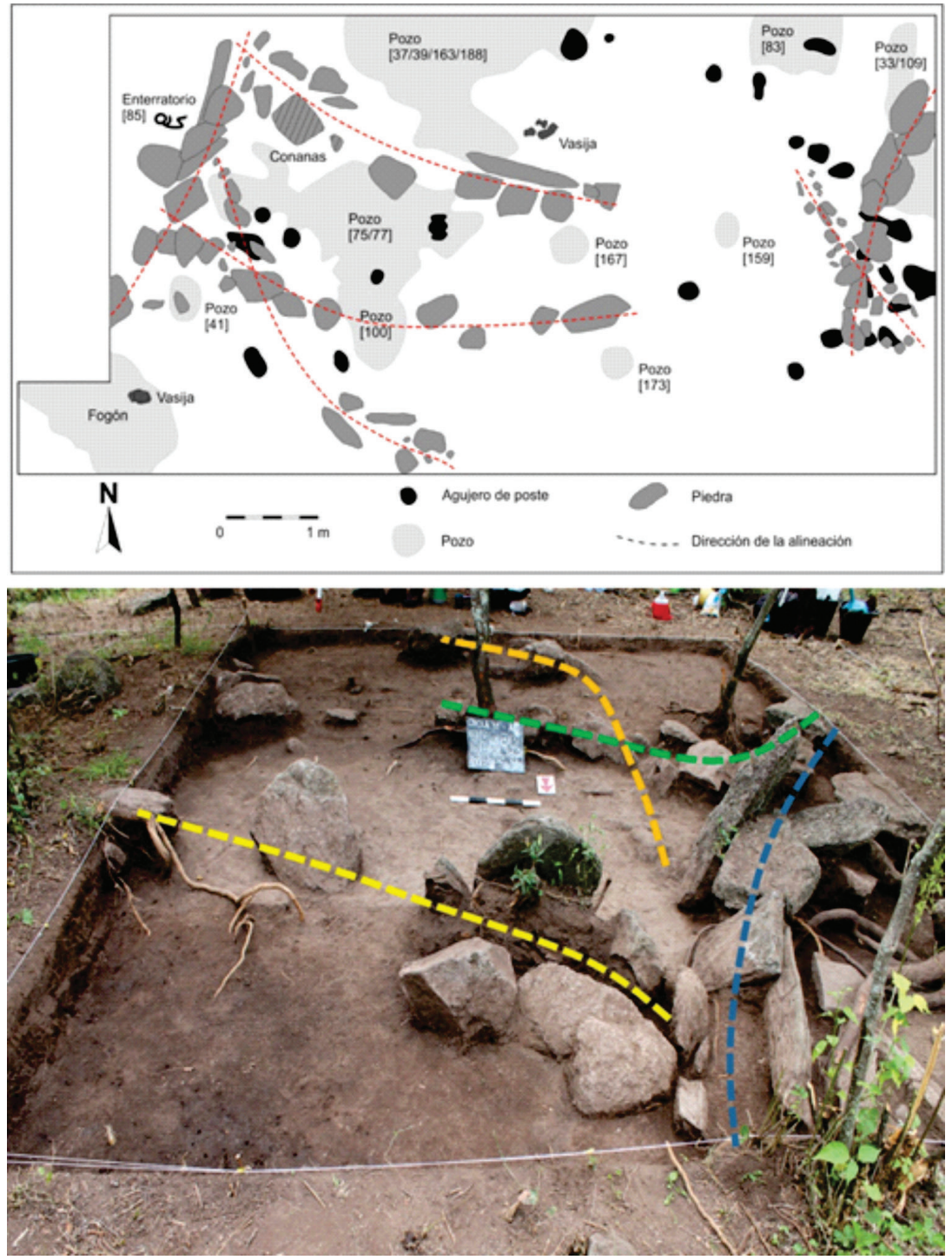

Figura 4. Arriba. Croquis de las alineaciones de rocas que conforman el sitio Oy31. Abajo. Vista de algunas de las alineaciones 
Sin embargo, por fuera del "Cerro de Oyola", pero en sus inmediaciones, el paisaje social es muy diferente, ya que se han registrado varios compuestos domésticos y espacios productivos, caracterizados por terrazas agrícolas ubicadas en la mayoría de las quebradas adyacentes a estos espacios arquitectónicos (Quesada et al. 2016; Zuccarelli 2020). De uno de estos compuestos, Oyola 50 (Oy50) proviene el último conjunto de materiales líticos analizados aquí (figura 5).

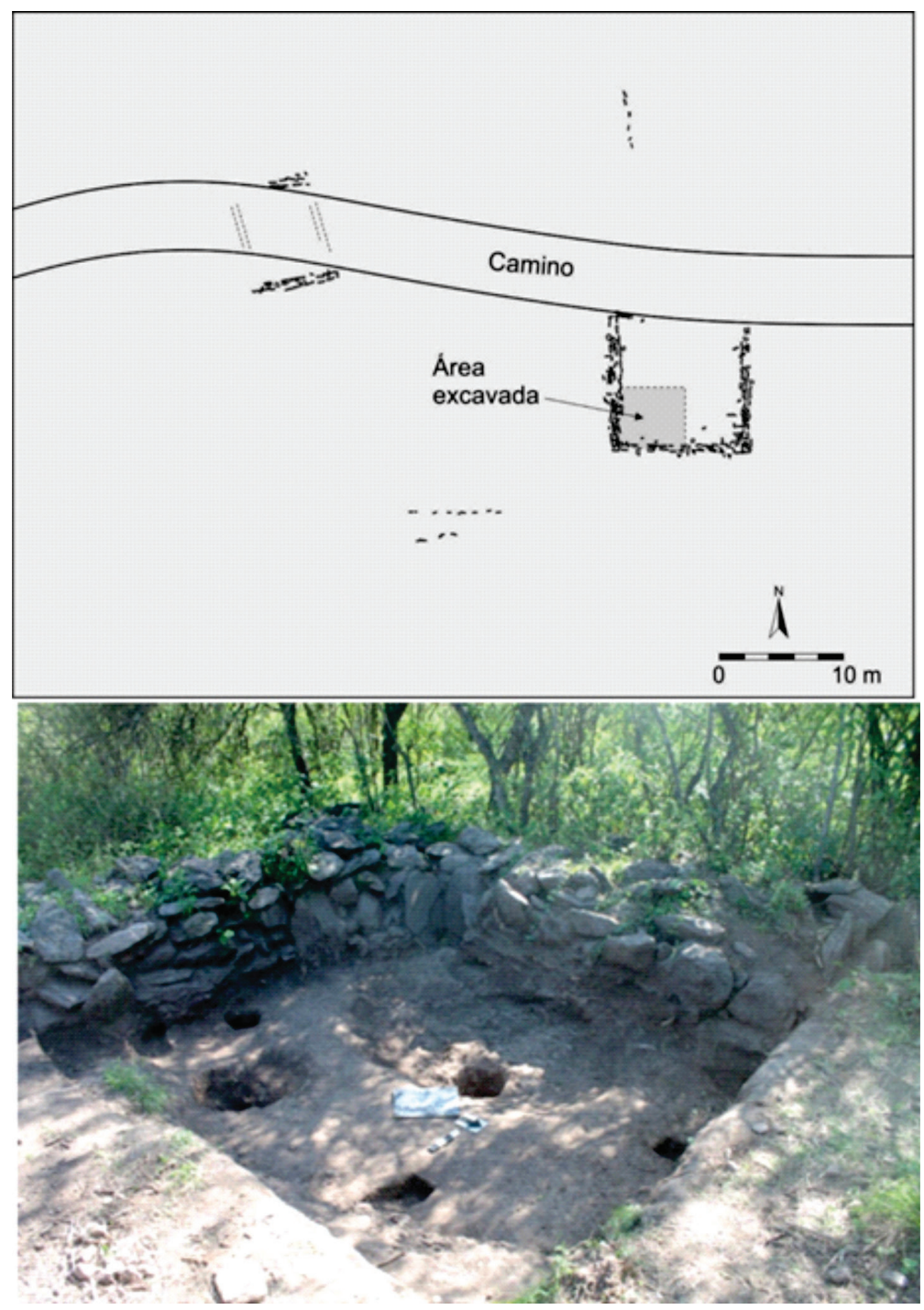

Figura 5. Arriba. Croquis del recinto excavado en Oy50. Abajo. Vista general de la excavación en Oy50

Este sitio está compuesto por muros de hasta $1 \mathrm{~m}$ de ancho y ha sido impactado por la construcción de un camino vecinal. Sin embargo, se puede observar un recinto cuadrangular, en regular estado de conservación, el cuál fue excavado parcialmente. La técnica constructiva usada en este sitio es similar a la de El Taco 19 (Quesada et al. 2012). Se destaca el grado de inversión de trabajo, tanto en el volumen de material utilizado en la construcción de los muros como en el cuidado en la técnica constructiva (Quesada et al. 2016; Quiroga Viñas 2020). La excavación 
estratigráfica de este sitio se ha concentrado en la esquina suroeste del recinto, ya que se trata del área mejor conservada de la estructura. Se la ha interpretado como una unidad doméstica, construida sobre un espacio donde se realizaron actividades previas a la construcción de los muros, como lo muestran las evidencias de actividades múltiples en unidades estratigráficas por debajo de la construcción de las paredes (Quiroga Viñas 2020). En este lugar, se habrían realizado, al igual que en ET19, la totalidad de la secuencia de producción lítica, tal como puede observarse por la presencia de diversas clases tipológicas y en donde se registra una coherencia en los tamaños de núcleos, desechos de talla e instrumentos manufacturados (tabla 1). Además, en un sector de esta estructura fueron identificados algunos bloques que parecen haber sido utilizados como yunques, ya que presentan huellas de percusión, asociados con percutores y desechos de talla (Egea et al. 2019; Quiroga Viñas 2020).

Los fechados para estos cuatro sitios muestran una fuerte sincronía (figura 6), más allá de las claras diferencias en sus características constructivas y de modalidad de ocupación.

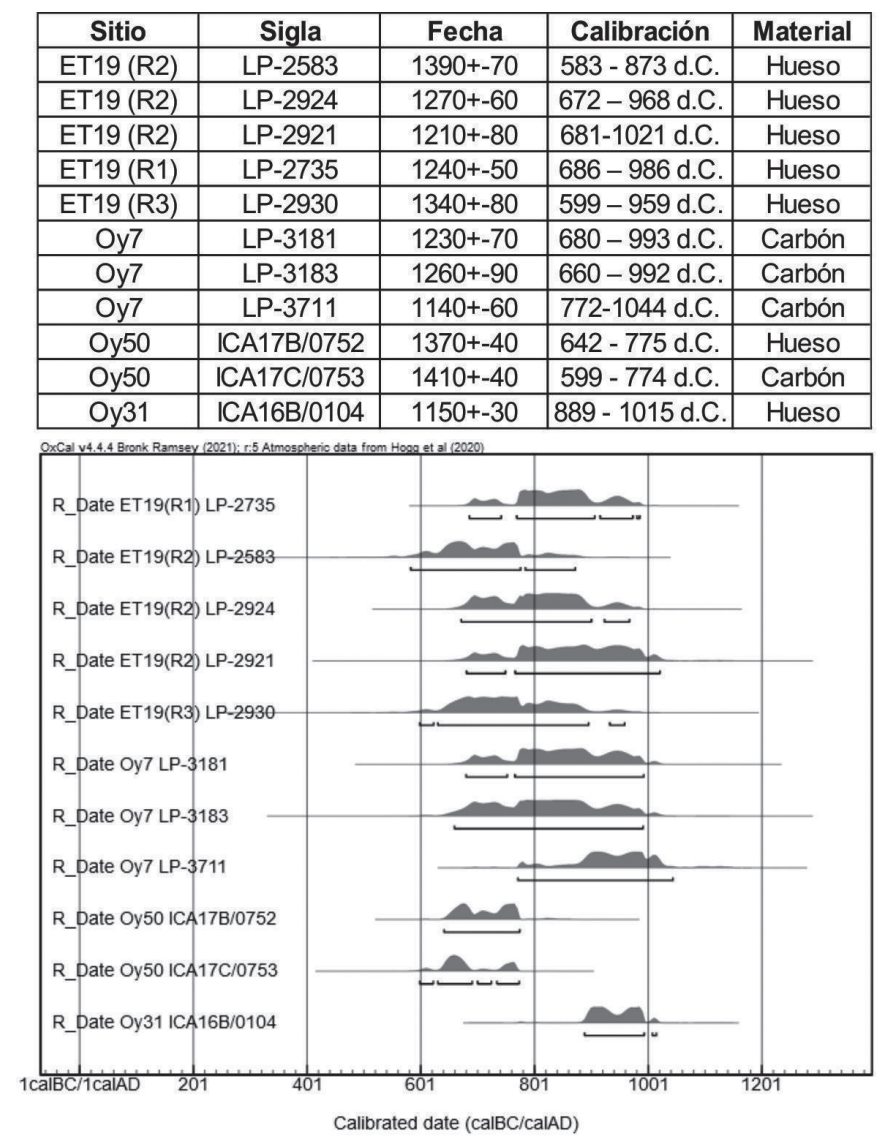

Figura 6. Fechados radiocarbónicos calibrados (Hogg et al. 2020) realizados en los cuatro sitios analizados

Vale aclarar aquí que se identificaron fuentes de aprovisionamiento de cuarzo a pocos metros de todos los sitios trabajados hasta el momento, con un máximo de unos $500 \mathrm{~m}$ de distancia. En el caso de El Taco, en la zona cumbral y en cercanías de ET19 se identificaron afloramientos de cuarzo pegmatítico de grandes dimensiones, donde hemos registrado desechos de talla, núcleos y percutores, lo que indicaría la realización de actividades de tallado y/o testeo en el lugar. Además, 
se observan nódulos de tamaños y formas muy diversos (Moreno 2015). Para el caso de Oyola, la presencia de este mineral es más reducida y no se han observado evidencias de su utilización en el pasado, pero esto puede deberse también a la baja o casi nula visibilidad de este paisaje, principalmente debido a la espesa vegetación. En general, se observan bloques pequeños dispersos (Egea 2016). Sin embargo, más allá de esta diferencia entre ambos sectores, que consideramos relevantes tal como veremos más adelante, el cuarzo es conspicuo en todo el paisaje de la Sierra de El Alto-Ancasti y por lo tanto su acceso puede considerarse simple.

\section{METODOLOGÍA}

Para el estudio de la tecnología lítica tuvimos en cuenta la propuesta desarrollada por Aschero $(1975,1983)$ y algunas modificaciones planteadas por otros autores (Aschero y Hocsman 2004), pero también, consideramos algunas cuestiones específicas vinculadas a la tecnología lítica en cuarzo, como las variedades utilizadas o algunos rasgos diagnósticos específicos para esta materia prima (Mourre 1996; Fábregas Valcarce y Rodríguez Rellán 2008; Driscoll 2011; Pautassi y Sario 2014; Sario y Pautasi 2015; Egea 2016; Pautassi 2018).

Específicamente, nos concentramos en aquellos aspectos vinculados al estudio de los instrumentos formatizados. En este sentido, la metodología se centró en dos grandes aspectos: por un lado, la caracterización de las formas base y, por el otro, la formatización de los biseles. En relación con el primer aspecto, tuvimos en cuenta los caracteres básicos de la forma base, que nos permiten diferenciar si se trata de una lasca, nódulo, núcleo, etc. Como, la muestra analizada posee principalmente formas base lasca, evaluamos el tipo de lasca para comprender las etapas de reducción de núcleo comprometidas en la obtención de las formas base y para acercarnos a las técnicas de reducción utilizadas. También tuvimos en cuenta, la fragmentación y aspectos vinculados con las dimensiones de los instrumentos, como tamaño, módulo y espesor tanto en medidas relativas como absolutas. Se utilizó el gráfico de Bagolini para la asignación de tamaños y módulos de longitud-anchura (Aschero 1975), mientras que en el caso de los espesores se utilizaron medidas absolutas de espesor máximo, estableciendo las siguientes categorías: muy delgado, de 0 a $5 \mathrm{~mm}$; delgado, de 5,1 a $10 \mathrm{~mm}$; espeso, de 10,1 a $20 \mathrm{~mm}$ y muy espeso de más de 20,1 mm. Todas las medidas fueron tomadas en mm con la utilización de un calibre digital.

La caracterización de los biseles activos se centró en la cantidad de filos presentes por espécimen, el largo del filo -teniendo en cuenta su longitud en relación con el tamaño general de la pieza-, la forma del bisel -simétrico o asimétrico-, su ángulo -expresado en grados-, la situación de los lascados - unifacial o bifacial-, la serie técnica-extensión de los lascados sobre las caras, indicando si son marginales, parcialmente extendidos o extendidos- y la forma de los lascados -escamosos, paralelos, escalonados, etc.-.

Finalmente, se tuvo en cuenta la asignación de los filos al grupo tipológico correspondiente (Aschero 1975, 1983) tendiente a avanzar en la interpretación funcional. Los instrumentos de cuarzo presentan ciertas dificultades para diferenciar aspectos tecnológicos registrados en la propuesta de Aschero (1975) ya que los rasgos diagnósticos producto de la talla resultan poco visibles (de Lombera Hermida 2009; Driscoll 2009; Moreno 2015; Pautassi 2018). Esto se debe a ciertas propiedades mecánicas de la materia prima, como la dureza y la presencia de planos de fractura e inclusiones (Pautassi y Sario 2014; Sario y Pautassi 2015; Egea y Gerola 2020; Moreno y Egea 2020; entre otros). Asimismo, los artefactos realizados en este mineral suelen tener menos estandarización morfológica que los elaborados con otras materias primas (Mourre 1996; de Lombera Hermida 2009; Driscoll 2011).

En este sentido, y teniendo en cuanta estas dificultades, se engloban grupos tipológicos vinculados a su potencial funcionalidad, centrados en algunas variables específicas. En este punto 
realizamos una asignación funcional de los filos registrados de acuerdo a su potencial uso en el pasado, considerando el largo del filo, la forma y el ángulo. El objetivo es generar grandes categorías, ya que, debido a su manufactura simple, estos instrumentos suelen ser registrados como instrumentos de formatización sumaria o lascas con retoques, pero creemos que de esta manera se puede alcanzar un agrupamiento que nos acerque de mejor manera a las prácticas sociales del pasado.

De esta manera, siguiendo lo planteado por Aschero $(1975,1983)$, pero ajustando la caracterización para tratar de unificar criterios funcionales ${ }^{1}$ (Moreno et al. 2021), entendemos por instrumentos de corte aquellos que presentan filos largos simétricos, normales, regulares y ángulos agudos menores de $50^{\circ}$. Proponemos la unificación en esta categoría de todos aquellos potenciales instrumentos vinculados a actividades de corte, como cortantes, cuchillos con retoque y filos naturales con rastros complementarios. Esto se plantea para priorizar la identificación de rasgos generales que nos acerque a las funciones de los instrumentos, en vez de centrarnos en caracteres tecnológicos que son muy difíciles de registrar en cuarzo.

Los raspadores presentan filos asimétricos, normales, continuos, con ángulos abruptos u oblicuos, superiores a $70^{\circ}$ y poseen un frente más o menos redondeado, en general, unifacial. Las raederas tienen un bisel asimétrico, ángulos medios $\left(50^{\circ} \mathrm{a} 70^{\circ}\right)$, con retoques continuos y regulares que determinan un filo largo con borde recto o semiconvexo. Las muescas son instrumentos de filo restringido, con una escotadura entrante intencional y definida, de diversas profundidades y que pueden ser de lascado simple o retocadas. Las puntas de proyectil, por su parte, son artefactos generalmente bifaciales, retocados, con bordes convergentes rectilíneos, presentan sección longitudinal simétrica y un extremo terminado en punta. Algunas poseen pedúnculo destacado y aletas entrantes agudas, mientras que otras presentan forma lanceolada. Los perforadores son instrumentos con punta destacada de terminación aguda determinada por retoques. Finamente, los filos naturales con rastros complementarios (FNRC) se producen durante el contacto del bisel no retocado sobre otros materiales, como cuero, carne, hueso o madera.

El conjunto lítico analizado se encuentra conformado por un total de 4.452 materiales líticos provenientes de los cuatro sitios referidos con anterioridad (tabla 1). En ET19, el total de materiales líticos tallados es de 1.539 (34,6\% del conjunto total), Oy7 aportó 1.125 (25,3\%), Oy31 302 $(6,8 \%)$ y Oy50 $1.489(33,4 \%)$. En todos los casos, alrededor del 96\% del conjunto fue manufacturado en cuarzo, aunque están presentes, en porcentajes muy reducidos, materias primas como sílice, cuarcita, filita, ópalo y basalto. A continuación, realizaremos una breve caracterización del conjunto de instrumentos, para luego introducirnos en las semejanzas y diferencias encontradas entre los distintos contextos trabajados. ${ }^{2}$

\section{LOS INSTRUMENTOS LÍTICOS DE CUARZO EN LA SIERRA DE EL ALTO-ANCASTI}

Cuando analizamos los instrumentos manufacturados recuperados en todos los conjuntos, lo primero que llama la atención es su bajo porcentaje (alrededor del 7\%) en relación con los desechos de talla $(92,9 \%, \mathrm{n}=4.137)$. Se registró un total de 306 instrumentos en los cuatros sitios excavados. En cuanto a la materia prima utilizada, se repite lo evidenciado en el conjunto general, es decir, la utilización del cuarzo, aunque en un porcentaje un tanto menor que lo evidenciado en el conjunto general $(90,8 \%, \mathrm{n}=278)$. Esto implica que habría habido un relativo, aunque esporádico, interés en seleccionar materias primas para la preparación de instrumentos formatizados, y nos permite pensar que algunos instrumentos de otras materias primas fueron ingresados ya manufacturados a los sitios, dado que no se registraron o se registraron en escasísimos porcentajes, desechos de talla de estas materias primas.

Aunque esto no será tema de discusión en este trabajo, cabe mencionar brevemente estas materias primas que también fueron utilizadas para confeccionar instrumentos. Hay en el 
conjunto un total de 28 instrumentos que no fueron confeccionados en cuarzo: 9 en filita, 3 en cuarcita y 16 en otras rocas silíceas (Figura 7). La filita fue seleccionada en todos los casos para la confección de cuchillos. Se trata de láminas de filita que presentan retoques sobre el bisel y que habrían permitido la realización de acciones de corte con ángulos muy agudos, menores a $20^{\circ}$. Este tipo de instrumentos ha sido registrado en otros contextos del NOA (Carbonelli 2011; Pazzarelli 2011, 2013; Sentinelli 2012; Gaál 2014; Franco Salvi et al. 2016; Montegú 2018). Creemos que la elección de esta materia prima para este tipo de instrumentos se sustenta en la dificultad técnica del cuarzo para obtener formas base con biseles tan agudos. Por otro lado, seis puntas de proyectil (35\% del total de puntas) fueron confeccionadas en materias primas distintas al cuarzo (una en cuarcita y cinco en otras materias primas todavía no identificadas). Además, hay una lasca con filo natural con rastros complementarios en cuarcita, una raedera y 10 instrumentos de corte en rocas silíceas. La distribución de estos grupos tipológicos entre las distintas materias primas del conjunto, muestra que en El Alto-Ancasti las personas que tallaban preferían ciertas materias primas para confeccionar determinados tipos de filos, como sería el caso de la filita para los cuchillos y la cuarcita para algunas puntas de proyectil. Esto probablemente se vincule con la dificultad de obtener formas base apropiadas para manufacturar este tipo de instrumentos en las variedades de cuarzo presentes en el área. No contamos con información de las posibles fuentes de aprovisionamiento de estas materias primas, pero en todas las prospecciones realizadas en la zona no han podido ser identificadas.

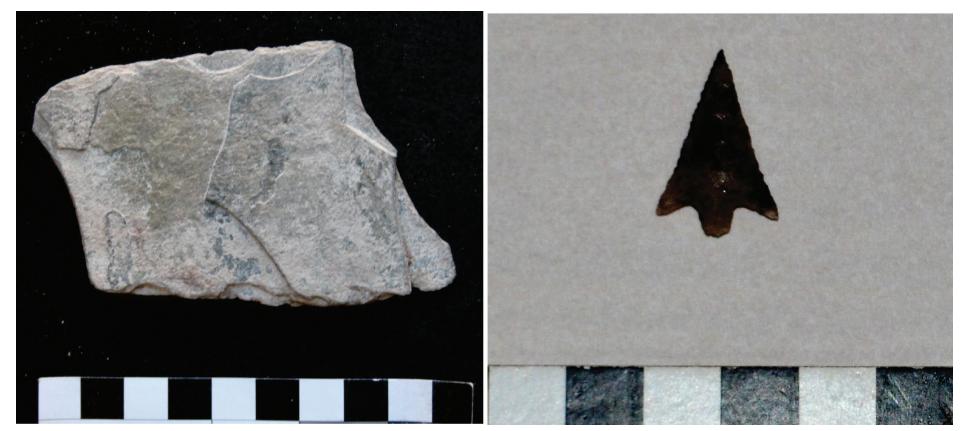

Figura 7. Ejemplo de instrumentos confeccionados en materias primas diferentes al cuarzo. A la izquierda, cuchillo de filita y a la derecha, punta de proyectil en roca silícea

Retornando al interés principal de este trabajo, que son los instrumentos manufacturados en cuarzo, en primera instancia nos centraremos en las características de las formas base registradas para la preparación de instrumentos (tabla 2). Se observa un amplio predominio de formas base lascas (86\%), mientras que el 14\% restante corresponde a formas base no diferenciadas. Entre las formas base lascas, predominan las lascas angulares y, a pesar del fuerte predominio de estas, se registran otros tipos como lascas de arista, planas y no diferenciadas. Los porcentajes para cada tipo de lasca son compatibles con lo observado en el conjunto de desechos de talla (Egea 2016; Moreno 2015) y puede tener un fuerte correlato con las dificultades en la reducción de los núcleos y la obtención de formas bases más estandarizadas o intencionalmente deseadas (Moreno y Egea 2020). Por el contrario, creemos que se habrían seleccionado formas base con características básicas necesarias y que justificaran su utilización para la formatización del filo. Nos referimos principalmente al largo del bisel, ángulo, tamaño y espesor de la forma base plausible de ser sostenida manualmente para realizar la acción. 
D. EGEA Y E. MoRENo - INSTRUMENTOS Líticos de CUARZo, PRÁCTICAS SOCIALES Y VIDA CAMPESINA DURANTE ...

Tabla 2. Comparación de las variables analizadas en relación con la manufactura de los filos líticos identificados en los sitios considerados

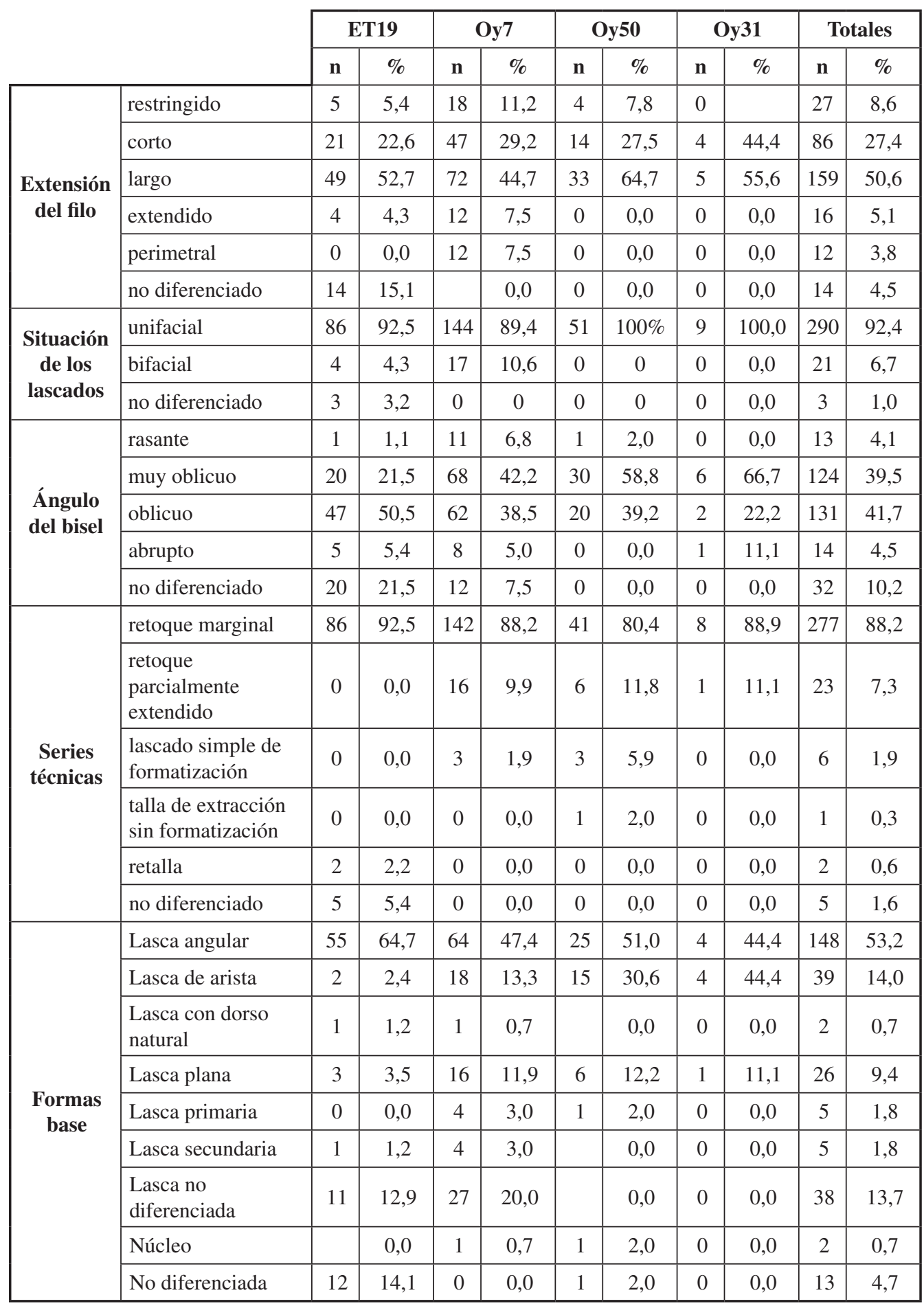


El porcentaje de fragmentación de los instrumentos es del 50,9\%, bastante menor que el $61,5 \%$ registrado entre los desechos de talla. En relación con los tamaños, se observan una mayor representación de los mediano pequeños y pequeños frente a aquellos grandes y muy grandes. En cuanto a los módulos de longitud-anchura, hay una gran dispersión, pero con muy baja representación de los valores extremos, tanto en ancho como en largo. Esto se vincula, además, con una tendencia de formas base cuadrangulares. Si tomamos en cuenta las medidas absolutas, la mayor cantidad de formas base oscila entre 10 y $35 \mathrm{~mm}$, tanto de ancho como de largo total. Finalmente, en relación con los espesores, notamos una tendencia bastante equilibrada entre los distintos valores de espesor (figura 8). Como se verá más adelante, esto tiene relevancia al cruzar la asignación de los grupos tipológicos identificados en el conjunto, tal como fuera adelantando en otra oportunidad (Egea 2018).
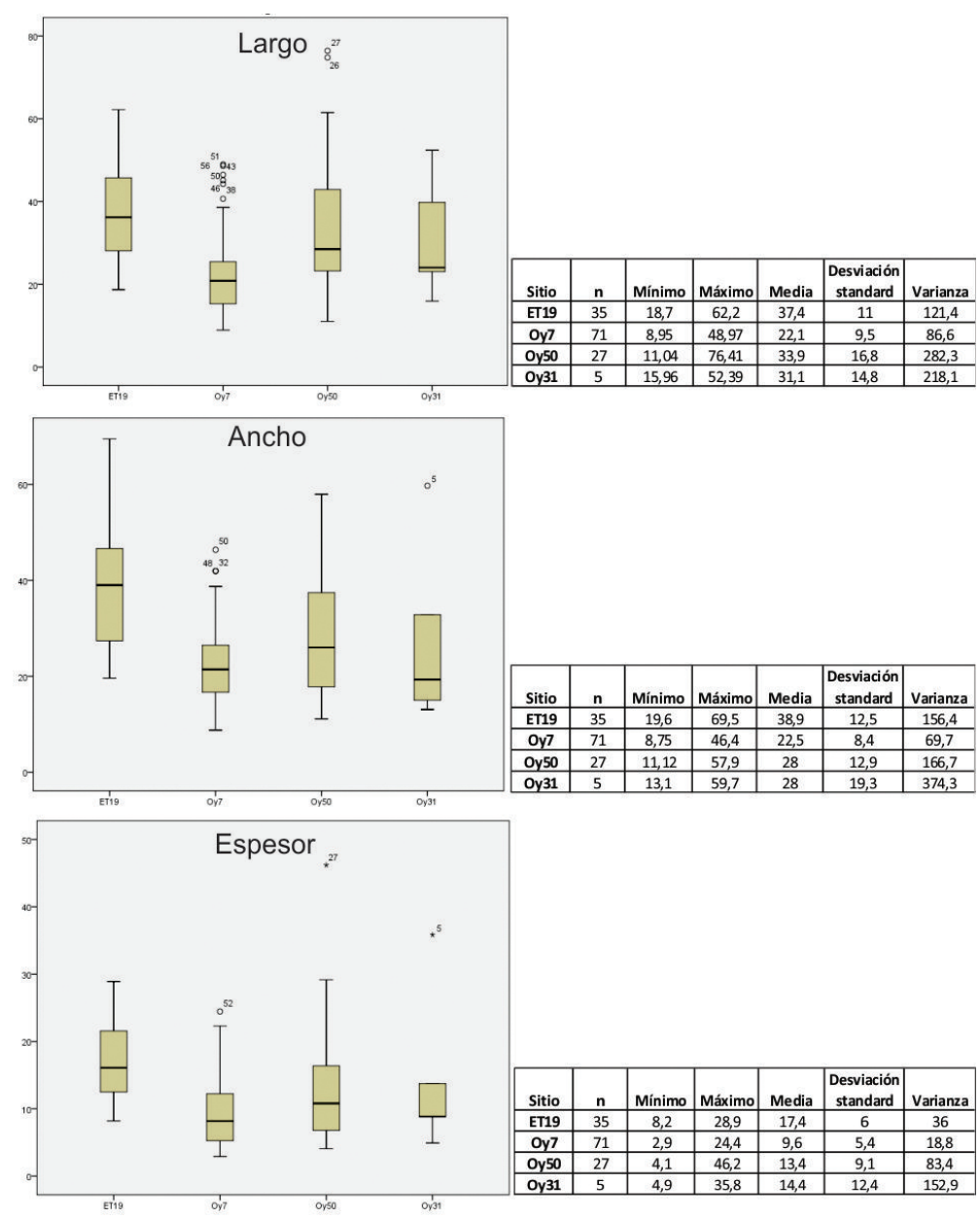

Figura 8. Gráficos que indican los valores de largo, ancho y espesor, medidos en mm, de los instrumentos manufacturados en cada uno de los sitios analizados

En relación con la articulación entre el tamaño y el espesor, se observa cierta correlación entre piezas espesas y de mayor tamaño. Esto se ha vinculado (en la utilización de talla a mano alzada) con la elección tecnológica de realizar el golpe hacia el interior de la plataforma de 
percusión, porque de esta manera, se aumentan las posibilidades de traspasar los planos de fractura y obtener formas base de mayor tamaño (Tallavaara et al. 2010). Sin embargo, esto implica, a su vez, menor control del golpe, ya que aumenta la fuerza necesaria para producir la fractura. Algunos autores han planteado esta misma característica en la utilización de la talla bipolar (Prous 2004; Pautassi y Sario 2014; Pautassi 2018), aunque es posible que haya menor espesor en las formas base obtenidas por talla bipolar debido a la diferencia en el tamaño del núcleo utilizado en ambas técnicas de reducción (Moreno y Egea 2020). Esta podría ser otra de las razones de la ausencia de estandarización en las formas base en cuarzo, independientemente de la técnica de talla utilizada.

\section{Filos, puntas y superficies}

En el conjunto general de instrumentos líticos, se observa que, de los 278 instrumentos de cuarzo recuperados, 27 presentan dos o más filos; correspondientes a 11 instrumentos compuestos dobles y 16 instrumentos no compuestos. ${ }^{3}$ Más adelante se evaluará en detalle cuáles son las combinaciones en estos casos.

El análisis general de los filos manufacturados en cuarzo muestra una escasa inversión de trabajo, evidenciada por el predominio de las series técnicas marginales (el 92\% del conjunto fue confeccionado de esta manera) (tabla 2). Además, la gran mayoría de los biseles son unifaciales. Se registran principalmente en posición unifacial directa (46\%), seguido de un porcentaje moderado (33\%) de unifacial inverso y una pequeña proporción (17\%) de unifacial no diferenciado. La bifacialidad también se encuentra presente, pero solo en un porcentaje muy menor de los instrumentos manufacturados.

En cuanto a los grupos tipológicos, o tipos de instrumentos presentes, vemos una amplia variedad: instrumentos de corte $(45 \%)$, raspadores $(22 \%)$, muescas $(15 \%)$, raederas $(10 \%)$, puntas de proyectil $(2,9 \%)$, artefactos no diferenciados $(4 \%)$, FNRC $(0,6 \%)$ y perforador $(0,3 \%)$ (figura 9 y tabla 1$)$.

La mayor parte de los filos del conjunto (77\%) -las raederas, cortantes, raspadores y cuchillos- permiten un rango de acciones generalizadas y habilitan funciones de raspado de superficies menos restringidas y de cortes largos o continuos (sensu Escola 2000). Esto podría relacionarse, también, con que la mayoría de los instrumentos presenta una extensión de los filos activos largos $(50,6 \%)$ que pueden ajustarse a un rango amplio de acciones.

Otro grupo menor (19\%) son filos específicos, que permiten una relativa especialización para determinado tipo de funciones. Las muescas, por ejemplo, habrían cumplido las acciones de raspado o desbastado en superficies convexas restringidas. Otro grupo tipológico específico, pero asociado a otras acciones, corresponde a las puntas de proyectil.

En cuanto a los instrumentos compuestos, ocho de los once compuestos dobles incluyen la representación de muescas en las combinaciones de grupos tipológicos, cuatro casos corresponden a muesca/cortante, tres a muesca/raedera y uno a muesca/raspador. Se ha registrado, además, un cortante/raedera y dos casos de cortante/raspador. Se da un solo caso de un instrumento compuesto múltiple que exhibe tres filos correspondientes a cortante y dos muescas. Por otro lado, hay dieciséis filos no compuestos dobles, estos son: nueve cortantes dobles, una raedera doble, una muesca doble y cinco raspadores dobles (tabla 3 ).

Los ángulos de los biseles son principalmente oblicuos (de $45^{\circ}$ a $65^{\circ}$ ) correspondientes sobre todo a raspadores y raederas, y muy oblicuos (de $30^{\circ}$ a $44^{\circ}$ ), pertenecientes a instrumentos de corte. Los ángulos rasantes y los abruptos se registran en porcentajes pequeños (tabla 2).

En resumen, si evaluamos tanto las características generales de las formas base como de los filos manufacturados, identificamos una variabilidad alta, que no demarca elecciones sistemáti- 


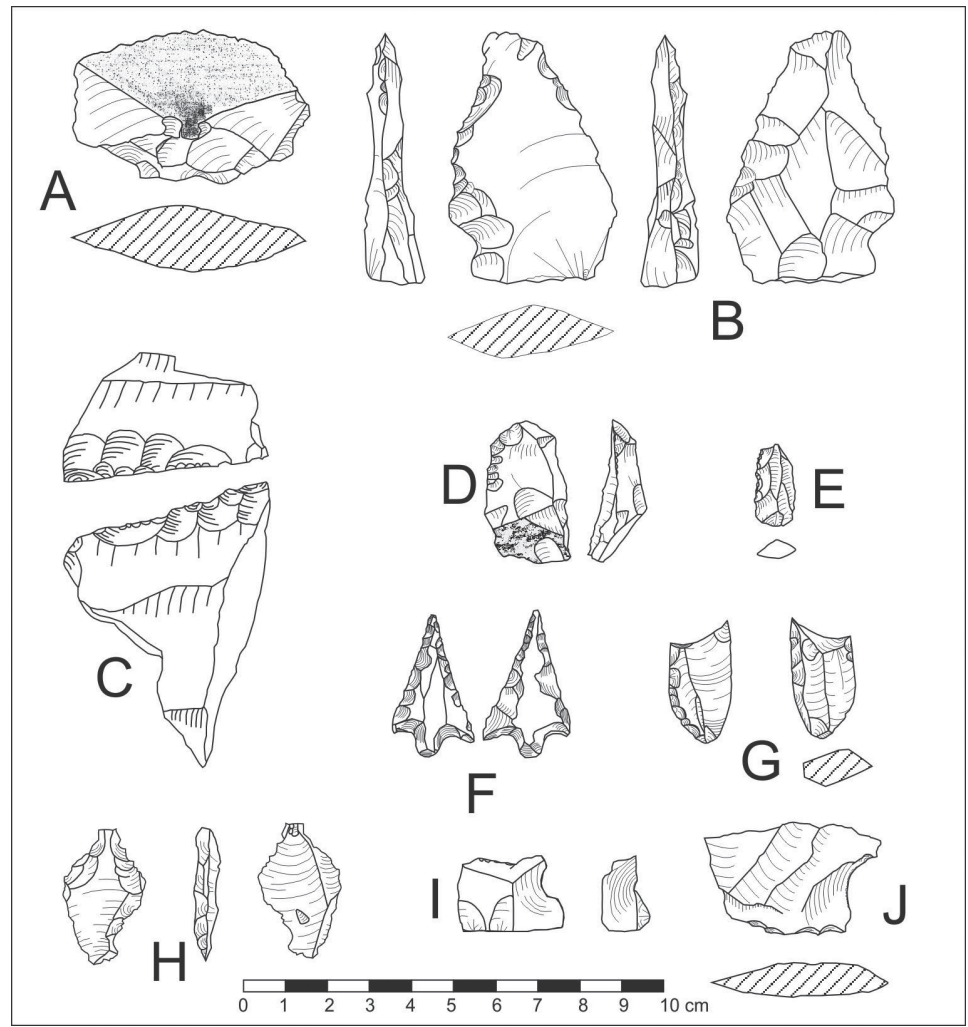

Figura 9. Instrumentos en cuarzo. A) Cortante de filo natural. B) Instrumento compuesto por un filo tipo cortante y un filo tipo raspador. C) Raspador de gran tamaño y ángulo abrupto. D y E) ejemplos de raederas. F) Punta de proyectil con pedúnculo. G) Fragmento de instrumento bifacial no diferenciado.

H) Perforador fracturado. I y J) Muescas. La última presenta la muesca más abierta registrada

Tabla 3. Representación de instrumentos compuestos y no compuestos por sitio arqueológico analizado

\begin{tabular}{|c|c|c|c|c|c|c|}
\hline & \multicolumn{6}{|c|}{ Compuestos } \\
\hline & $\mathbf{N}$ & $\%$ & ET19 & Oy7 & Oy50 & Oy31 \\
\hline Muesca/cortante & 4 & 36,4 & 0 & 3 & 1 & 0 \\
\hline Muesca/raedera & 3 & 27,3 & 1 & 2 & 0 & 0 \\
\hline Muesca/raspador & 1 & 9,1 & 0 & 1 & 0 & 0 \\
\hline Cortante/raedera & 1 & 9,1 & 1 & 0 & 0 & 0 \\
\hline Cortante/raspador & 2 & 18,2 & 1 & 1 & 0 & 0 \\
\hline \multirow[t]{3}{*}{ Total } & 11 & 100 & 3 & 7 & 1 & 0 \\
\hline & \multicolumn{6}{|c|}{ No compuestos } \\
\hline & $\mathbf{N}$ & $\%$ & ET19 & Oy7 & Oy50 & Oy31 \\
\hline Cortante doble & 9 & 56,3 & 0 & 8 & 1 & 0 \\
\hline Raedera doble & 1 & 6,3 & 0 & 1 & 0 & 0 \\
\hline Muesca doble & 1 & 6,3 & 1 & 0 & 0 & 0 \\
\hline Raspador doble & 5 & 31,3 & 1 & 4 & 0 & 0 \\
\hline Total & 16 & 100 & 2 & 13 & 1 & 0 \\
\hline
\end{tabular}


cas en cuanto a tamaño, forma o espesor de la forma base (Moreno y Egea 2020). Esto podría vincularse a la dificultad para controlar la fractura del cuarzo y a la presencia de gran cantidad de inclusiones, planos de fractura y otros elementos internos de esta materia prima. De esta manera, creemos que la obtención de formas base que cumplieran con caracteres básicos aptos para la manufactura de filos, habría sido la clave implementada por los pobladores y las pobladoras del este catamarqueño. En el mismo sentido, la manufactura general del conjunto presenta caracteres simples, tendientes a la realización de múltiples acciones que requiriesen mayoritariamente filos largos, de ángulo variable y tamaños que permitieran la prensión manual (Moreno 2015).

Esta relación de las poblaciones humanas del este catamarqueño con el cuarzo utilizado para la preparación de instrumentos podría estar signada por la simpleza, por la falta de sistematicidad o de pautas artesanales en la reducción de nódulos y núcleos y en la formatización de los biseles. Sin embargo, creemos que esta tecnología se vincula con las formas de construcción de relaciones entre estas personas y el entorno local, en pautas de aprendizaje vinculadas a las formas de reducción del cuarzo y a la preparación de instrumentos específicos de acuerdo a las necesidades y, sobre todo, a un marco en el cual se construían los mundos de la vida en torno a la vida social cotidiana. Además, creemos que si miramos un poco más en detalle las similitudes y diferencias entre los sitios trabajados, podemos profundizar en algunos de estos aspectos.

\section{TECNOLOGÍA LÍTICA EN LOS DIFERENTES ESPACIOS INVESTIGADOS EN LA SIERRA DE EL ALTO-ANCASTI.}

A continuación, se presentan brevemente las características generales de los conjuntos de instrumentos de cada uno de los sitios trabajados para, luego, hacer una comparación en la cual podamos analizar la forma en que se construyeron las relaciones de las personas con la tecnología lítica de manera diferencial en cada uno de estos lugares.

\section{El Taco 19}

El Taco 19 es un sitio habitacional doméstico que, en relación con su historia ocupacional, muestra una dinámica de transformaciones arquitectónicas y cambios de uso de los recintos que lo conforman; sin embargo, esto no implica necesariamente pausas en su ocupación (Quesada y Gastaldi 2013; Ahumada et al. 2019). Se interpretó que en este sitio se realizaron múltiples actividades de reproducción doméstica, entre las cuales las herramientas líticas habrían jugado un importante rol. El conjunto lítico recuperado consta de 1.434 desechos de talla, 85 instrumentos, 15 núcleos de cuarzo y 5 percutores también de esta materia prima (tabla 1). La muestra analizada para este trabajo está conformada por 93 filos líticos de cuarzo, que se corresponden con 85 instrumentos. Los grupos tipológicos presentes son raspadores, muescas, raederas, instrumentos de corte, no diferenciados, instrumentos bifaciales no diferenciados, FNRC y un perforador. En el caso de los instrumentos bifaciales, se trata de cuatros fragmentos que presentan un bisel retocado en ambas caras. Sin embargo, no han podido asignarse a un grupo tipológico ya que presentan fracturas sobre el filo retocado. Es posible que estas fracturas se hayan producido durante el proceso de manufactura. Hay tres instrumentos con filos dobles en el conjunto, un raspador doble, una muesca doble y un instrumento bifacial no diferenciado. En el caso de los instrumentos múltiples se trata de combinaciones de cortante/muesca, cortante/ raedera, y raedera/muesca (tabla 1).

Las formas base usadas para confeccionarlos comprenden principalmente lascas, al igual que en el conjunto general, mayoritariamente angulares. El índice de fragmentación de los instrumentos es relativamente alto (tabla 1). 
En cuanto a los tamaños, la mayoría se incluye en la categoría medianos, entre estos los mediano grande (38\%) y mediano pequeño (35\%) son los más representados. Los artefactos grandes constituyen el $22 \%$, el restante $5 \%$ está conformado por instrumentos de tamaño pequeño (4\%) y muy grande (1\%). Con respecto a los módulos de longitud-anchura se observa un equilibrio, resaltando levemente el mediano normal (35\%). Los espesores muestran la predominancia de la categoría espeso (64\%), seguida por proporciones menores de muy espeso (22\%) y delgado (14\%). Es el único sitio donde no hay espesores muy delgados (figura 8).

La mayoría de los filos son unifaciales, aunque se registraron algunos bifaciales. Al igual que en el conjunto general, la mayoría fueron confeccionados mediante retoque marginal. Si bien en todos los sitios prevalecen los ángulos muy oblicuos y oblicuos, en este caso sobresalen ampliamente los oblicuos sobre los muy oblicuos. En cuanto a la extensión de los filos, al igual que en todos los sitios de este conjunto, predominan los filos largos (tabla 2).

\section{Oyola 7}

En la cueva Oyola 7 se observa que el espacio ha sido ocupado y desocupado de forma cíclica. Aquí las actividades habrían estado relacionadas con visitas períodicas donde se habrían realizado actividades puntuales, posiblemente vinculadas al pintado de sus paredes (Gheco 2012, 2017; Gastaldi et al. 2016). El conjunto lítico de este sitio está conformado por 986 desechos de talla, 135 instrumentos y 4 núcleos de cuarzo. Los 135 instrumentos fueron manufacturados en cuarzo y dan cuenta de un total de 161 filos. Los tipos de filos corresponden a instrumentos de corte; raspadores, muescas, raederas y puntas de proyectil; además, hay filos no diferenciados (tabla 1). Se registraron trece artefactos dobles no compuestos (conformados por ocho cortantes dobles, una raedera doble, cuatro raspadores dobles) y nueve instrumentos compuestos (dados por las combinaciones de muesca/instrumento de corte, muesca/raedera, muesca/raspador e instrumentos de corte/raspador). También se registraron dos instrumentos múltiples, uno es una combinación de muesca doble e instrumento de corte, mientras que el otro es un instrumento de corte y otros dos filos que no pudieron ser diferenciados (tabla 3).

Las formas base usadas para la confección de estos instrumentos fueron casi en su totalidad lascas, principalmente angulares, aunque hay otros tipos de lascas (como las no diferenciadas, planas, de arista, secundarias y primarias). En un caso, también se registró el uso de núcleo como forma base. El porcentaje de fractura del conjunto es del $47 \%$ (tabla 2).

Los tamaños y espesores en este sitio son menores que en el resto de los casos analizados: prevalecen los tamaños pequeño (52\%) y mediano pequeño (33\%), seguidos por mediano grande (9\%), grande (3\%), muy pequeño $(2 \%)$ y muy grande $(1 \%)$; entre los espesores se destacan los delgados (44\%), seguidos de muy delgados $(25 \%)$ y espesos $(25 \%)$ en la misma proporción. Los módulos son muy variados, la mayoría mediano normal (36\%) y corto muy ancho (27\%) (figura 8).

En cuanto a los biseles, los ángulos son mayormente muy oblicuos y oblicuos. Los filos fueron realizados principalmente con retoque marginal, pero se registran también instrumentos con retoque parcialmente extendido y lascado simple de formatización. Las puntas de proyectil son bifaciales. Además, hay otros seis filos bifaciales, representación bastante mayor que la del conjunto general. La extensión de los filos es mayormente largo, aunque se registran filos cortos como restringidos, extendidos y perimetrales.

\section{Oyola 50}

Oyola 50, al igual que ET19, es un sitio habitacional doméstico, donde se ven ciertos cambios en el uso a lo largo de los aproximadamente 200 años en que fue ocupado. Además, ambos sitios 
se caracterizan por varios eventos de vertido de basura, que preparan superficies para nuevas actividades (Quesada et al. 2016; Quiroga Viñas 2020). El conjunto lítico presenta 1.428 desechos de talla, 49 instrumentos, 8 núcleos, 4 percutores y además se identificaron en el recinto dos piedras planas con marcas que habrían funcionado a modo de mesas de trabajo o yunques (Egea et al. 2019; Quiroga Viñas 2020). En este sitio se recuperaron un total de 49 instrumentos manufacturados en cuarzo y un total de 51 filos. Se registran cuatro grupos tipológicos: instrumentos de corte, muescas, raederas y raspadores (tabla 1). Hay dos instrumentos de filo doble, un cortante doble y un cortante/muesca (tabla 3 ).

Los instrumentos fracturados llegan al $45 \%$ del conjunto y las formas base usadas corresponden principalmente a las lascas de distintos tipos, con predominio de las angulares y un porcentaje relevante de lascas de arista, seguido de lascas planas y primarias (tabla 2).

Los tamaños presentes en el conjunto son mayormente medianos, tanto mediano pequeño (34\%) como mediano grande (26\%), seguidos de pequeño (19\%), grande (19\%) y muy grande (2\%). Los módulos y espesores son variados; los primeros se distribuyen entre mediano normal (51\%), corto ancho (20\%), mediano alargado (16\%), corto muy ancho (8\%), corto anchísimo (2\%) y laminar normal (2\%). Los espesores, por su parte, son delgados (44\%) y espesos (35\%) principalmente, seguidos de muy delgado (11\%) y muy espesos (10\%) (figura 8).

Los filos de este conjunto son en todos los casos unifaciales y prevalecen los largos, seguidos de cortos y restringidos. Los ángulos de los biseles son muy oblicuos, oblicuos y rasantes y fueron confeccionados con retoque, tanto marginal como parcialmente extendido (tabla 2).

\section{Oyola 31}

Finalmente, debido a la expeditividad de sus técnicas de construcción, la baja formalización de los espacios y las evidencias de reconstrucción y transformación de las plantas de las estructuras, Oyola 31 es interpretado como un lugar en el cual se prepararon campamentos transitorios, de uso eventual o poco prolongado (Quesada et al. 2016). El conjunto lítico de este sitio está conformado por 289 desechos de talla, 9 instrumentos y 4 núcleos de cuarzo. En este sitio, todos los artefactos fueron confeccionados en cuarzo. Los grupos tipológicos presentes son cortantes, raspadores, raedera, muesca y un instrumento no diferenciado (tabla 1). No hay filos dobles o compuestos en este conjunto.

El 44\% de los instrumentos se encuentra fracturado. Las formas base son lascas angulares y de arista en igual proporción. Los tamaños y módulos son variados con predominio de los tamaños mediano pequeño $(56 \%)$, mediano normal (44\%) y mediano alargado (44\%), mientras que los espesores son mayormente delgados (56\%) (figura 8).

Los filos son todos unifaciales, el 55\% de ellos es largo y fueron confeccionados con retoque tanto marginal como parcialmente extendido. Los ángulos de los biseles son muy oblicuos y oblicuos.

\section{COMPARACIÓN ENTRE LOS DIFERENTES SITIOS DE LA SIERRA}

Al evaluar las similitudes y diferencias entre los conjuntos de instrumentos manufacturados en cuarzo en los diferentes sitios investigados en la Sierra de El Alto-Ancasti, resaltan algunos aspectos generales que comentamos al principio, como el predominio de los filos unifaciales marginales manufacturados mediante retoque. Sin embargo, vemos algunos atributos que requieren una evaluación más profunda.

El primer punto a tener en cuenta es el porcentaje de instrumentos en relación con la cantidad total de materiales líticos en cada uno de los sitios. Allí, notamos un porcentaje bastante 
mayor en el caso de la cueva Oyola 7, que alcanza el $12 \%$ de instrumentos frente a la totalidad de materiales líticos, mientras que en los casos de ET19 (5,5\%), Oy31 (3,3\%) y Oy50 (3\%) son porcentajes considerablemente menores. Esta diferencia se vincula a la información obtenida de los conjuntos líticos donde se interpretó que, en los últimos sitios, se habría realizado la totalidad de la secuencia de producción, mientras que en el caso de Oy7 habrían ingresado los instrumentos ya manufacturados o formas base obtenidas en las vetas de cuarzo cercanas, que habrían sido formatizadas para la preparación de los filos utilizados (Moreno 2015; Egea 2016; Moreno y Egea 2016). En este sentido, dadas las características de la secuencia de momentos de mayor y menor intensidad de ocupación de la cueva (Gastaldi et al. 2016; Gheco 2017), consideramos que en su interior se habrían utilizado los instrumentos líticos manufacturados y que las actividades de talla habrían sido solo para formatización, por lo que la relación instrumentos/desechos de talla es relativamente mayor.

En este sentido, en el caso de Oyola 50 notamos la presencia de todas las etapas de producción, pero además, de empedrados a modo de mesas de trabajo, posiblemente utilizados como yunques para la talla bipolar; esto nos permite pensar que se generaron espacios destinados a estas tareas específicas. Por ello, en este sitio podemos asociar las prácticas de producción y mantenimiento con la idea de un taller lítico durante algún momento de su ocupación (Egea et al. 2019; Quiroga Viñas 2020).

Otra diferencia significativa es la tendencia a mayores tamaños y espesores en el conjunto de ET19 (figura 8), en relación con lo observado en los otros tres conjuntos. Esto podría explicarse, en parte, por su cercanía a una gran cantera de cuarzo (Moreno 2015), con nódulos de tamaños variables, que podría otorgar a los talladores la posibilidad de obtener una mayor variabilidad de formas base que las posibilidades brindadas en los casos de Oyola, donde las vetas de cuarzo son mucho más pequeñas, con nódulos generalmente de tamaño pequeño y, aunque numerosas en el paisaje, sin la distribución ubicua de las adyacencias de ET19 (Moreno 2015). Esto también se vincula con el espesor, que es sustancialmente mayor en el caso de ET19. De esta manera, resulta claro que la vinculación espacial con las canteras habría significado la posibilidad de desarrollar estrategias tecnológicas específicas y ciertas restricciones de diseño dado por los soportes presentes en cada caso.

Otro aspecto relevante en cuanto a la diferenciación entre los sitios son los grupos tipológicos presentes (figura 10). Prevalecen mayoritariamente los instrumentos de corte, salvo en el caso de ET19, donde son muy pocos (solamente el 18,9\%), mientras que en otros sitios (como en Oy50) alcanzan el 75\% del conjunto. Por el contrario, en ET19, la presencia de instrumentos es más equilibrada que en los demás sitios, con porcentajes relativamente similares de raspadores, muescas, instrumentos de corte y raederas (tabla 1). Esto podría vincularse a actividades específicas realizadas en cada uno de los sitios. Además, debemos tener en cuenta que en ET19 es donde se encuentra la mayor cantidad de instrumentos cortantes de filita, que podrían haber reemplazado a los instrumentos de corte manufacturados en cuarzo para algunas actividades (Moreno 2015). También, podría implicar que en ET19 la importancia de las actividades de raspado fue mayor, quizás, relacionadas a actividades de procesamiento secundario de animales -como el trabajo con cueros- vinculadas con el pastoreo de camélidos, actividad de relevancia en este sitio (Ahumada y Moreno 2015-16; Moreno y Ahumada 2018). Futuras investigaciones, quizás, puedan otorgar más conocimientos para su interpretación.

Adicionalmente, solo en ET19 se han registrado instrumentos bifaciales (exceptuando el caso de las puntas de proyectil en Oy7), sin embargo, no hemos podido asignarlos a un grupo tipológico debido a que se encuentran altamente fragmentados.

Los artefactos cortantes (sobresalientes principalmente en Oy50) posiblemente hayan sido utilizados en el procesamiento de animales para el consumo. Sin embargo, la variedad de instrumentos nos habla de diversas tareas relacionadas a la vida cotidiana, tal vez más diversificadas en 


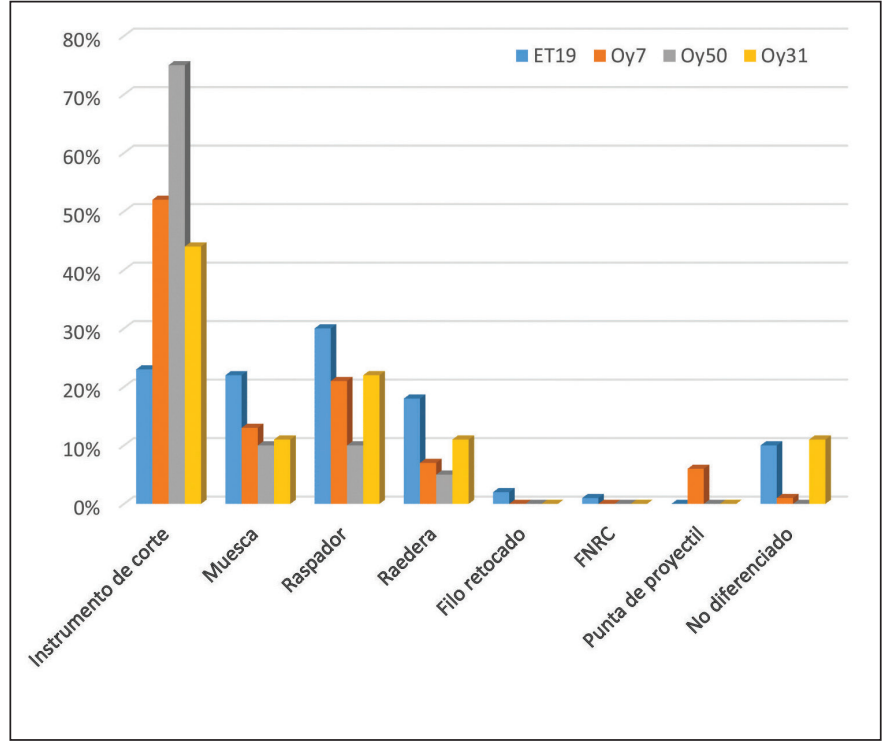

Figura 10. Comparación de los grupos tipológicos identificados en los diferentes sitios analizados

ET19 donde los grupos tipológicos son más y están representados de forma equilibrada. También podría significar que parte de las actividades se realizó en otro espacio externo u otro recinto con actividades específicas y que, por lo tanto, la menor diversidad artefactual sea consecuencia de esta situación. En el caso de ET19, al excavar los dos recintos (R1 y R2) se obtuvo información relevante sobre distintas prácticas, tanto vinculadas a la cocción y servicio de alimentos, como a otros tipos de actividades múltiples que se habrían llevado adelante principalmente en R2.

Finalmente, un punto significativo de diferencias entre los sitios es aportado por los instrumentos compuestos y no compuestos. Más allá de su presencia en todos los sitios (salvo en Oy31), notamos una gran importancia en el caso de Oy 7 , donde conforman casi el 15\% del total de filos manufacturados en el sitio. Esto podría implicar que en Oy7 ingresaron instrumentos de mayor versatilidad que aquellos manufacturados en los otros sitios. Posiblemente pueda vincularse a una mayor previsibilidad, donde los instrumentos habrían sido manufacturados con anticipación y posiblemente en otro lugar; en contraposición a los otros tres sitios, donde los instrumentos habrían sido preparados para la realización inmediata de alguna actividad específica. Sin embargo, consideramos que estudios experimentales y funcionales podrían otorgar más información sobre las posibles elecciones de los tipos de instrumentos presentes en cada caso.

Quizás, la diferencia más significativa en relación con los grupos tipológicos es la ausencia de puntas de proyectil tanto en ET19 como en Oy50 y Oy31. Esta situación es diferente en el caso de la cueva 7 de Oyola, donde se recuperaron catorce puntas de proyectil, de las cuales nueve fueron manufacturadas en cuarzo. Estas puntas de proyectil poseen un diseño bastante estandarizado: triangular pequeño, algunas con pedúnculo y otras apedunculadas de base escotada (figura 11). Esta situación de ausencia de puntas de proyectil es común en otros sitios excavados en la Sierra de El Alto-Ancasti, donde no se han recuperado en ningún contexto, aunque principalmente se han trabajado recintos interpretados como unidades domésticas (Nazar 2003; Dlugosz 2005; Gerola 2018), aunque hay algunos casos de cuevas con arte rupestre también excavadas en la zona, pero con ausencia de puntas de proyectil (Gerola 2018).

Esta situación es muy relevante, ya que no se registra la presencia de estos instrumentos, pero sí la de animales silvestres (cérvidos, zorros y posiblemente guanacos) en los conjuntos 
arqueofaunísticos, principalmente en ET19 (Ahumada y Moreno 2015-16; Moreno y Ahumada 2018). Antes del hallazgo de puntas de proyectil líticas en Oy7, se consideraba el posible empleo de otros mecanismos para las cacerías, como el uso de trampas o madera como materia prima para la preparación de las armas. Sin embargo, la información del conjunto de puntas de proyectil de Oy7 pone de relieve su presencia en la sierra de El Alto-Ancasti. Además, se observa un conocimiento acabado de la formatización de estos instrumentos, ya que exhiben retoque bifacial y una clara estandarización del diseño, más allá de la presencia o ausencia del pedúnculo. Sin embargo, resulta llamativo que en Oy7 no se haya recuperado información acerca del aprovechamiento de animales silvestres a través de la cacería, a pesar de ser un área donde se encuentran estas especies (Gastaldi et al. 2016; Moreno et al. 2019).

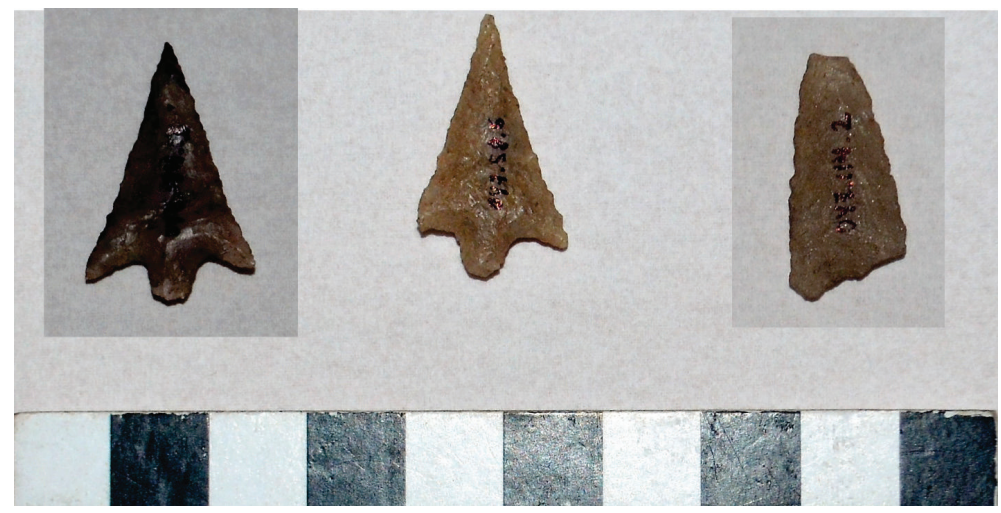

Figura 11. Puntas de proyectil en cuarzo recuperadas en Oy7

Las características de los desechos de talla recuperados en Oy7 no nos permiten asegurar que hayan sido manufacturadas en el interior de la cueva, ya que no se observan lascas de formatización compatibles con el tamaño de los lascados en las puntas de proyectil. Además, tampoco pudimos observar preformas en el conjunto analizado. Posiblemente, la presencia de las puntas de proyectil tenga que ver con algunas actividades específicas que se habrían realizado en los momentos de mayor actividad de Oyola 7, vinculados al encendido de fogones, la realización u observación de las pinturas rupestres y donde participaron estos instrumentos. En su mayoría, las puntas de proyectil se encuentran fracturadas, algunas de ellas con muy pequeñas fracturas en su ápice o en su base que habrían implicado necesariamente su mantenimiento o descarte. Solamente cuatro especímenes se encuentran completos. Esperamos que futuras investigaciones, sobre todo en otras cuevas con arte rupestre de la zona, puedan aportar información acerca de la presencia de las puntas de proyectil en estos espacios, o si se trata de una práctica singular en Oyola 7.

\section{REFLEXIONES FINALES}

Las poblaciones que ocuparon los diferentes pisos ambientales de la Sierra de El Alto-Ancasti se relacionaron de manera cotidiana con el cuarzo para la preparación de una multiplicidad de filos líticos con diversas funcionalidades. Así, pudimos observar algunas elecciones generales sobre las formas base utilizadas para la preparación de los instrumentos y las características básicas de la formatización de filos, vinculada al uso de retoque unifacial marginal, sin evidencias claras y sistemáticas de reactivación, lo que fuera definido por Escola (2000) como diseños utilitarios. Consideramos que esto se vincula a la dificultad para la talla que presenta el cuarzo -dada por su 
dureza y su estructura con gran cantidad de planos de fractura o inclusiones-aunque, sin embargo, resulta posible la manufactura por adelgazamiento y reducción bifacial, tal como se puede observar en el caso de las puntas de proyectil o en otros contextos del centro-norte argentino (Sario 2008; Pautassi 2018). Quizás, también se vincule a la necesidad de dar respuesta rápida a situaciones cotidianas y a la extrema cercanía de las fuentes de aprovisionamiento de esta materia prima. Sin embargo, debemos tener en cuenta que algunas de las diferencias observadas en el caso de ET19 pueden deberse a la existencia de canteras de cuarzo de gran tamaño con nódulos de tamaños diversos, que permitirían mayor flexibilidad a la hora de obtener formas base en contraposición a lo que ha sido registrado hasta el momento en las canteras ubicadas en Oyola.

Observamos también que la mayor parte de los instrumentos presentan filos largos y con múltiples funciones, además de altos porcentajes de fragmentación, lo que podría indicar las características de su utilización. Raspadores, raederas, instrumentos de corte y muescas son los filos más representados en el conjunto, que incluye algunos filos compuestos.

Ahora bien, cuando nos detenemos un poco más en las características de los conjuntos diferenciados entre los sitios registrados, notamos grandes semejanzas y algunas diferencias y es sobre estas últimas que nos queremos detener. En particular, creemos que el porcentaje de instrumentos en relación con el conjunto total de materiales líticos aporta a las historias de producción de estos materiales en cada uno de los espacios. En este sentido, el porcentaje más alto identificado en Oy7 fortalece la interpretación alcanzada en otras oportunidades acerca del ingreso a la cueva de instrumentos ya terminados o formas base que eran formatizadas en este lugar (Egea 2016; Moreno y Egea 2016). Por el contrario, en ET19 y en Oy50 es más clara la presencia de toda la secuencia de producción lítica, marcada por el hallazgo de núcleos, percutores, desechos de talla, e incluso de posibles yunques utilizados para la realización de talla bipolar (Moreno 2015; Quiroga Viñas 2020). Por su parte, el pequeño conjunto de Oy31 no permite alcanzar conclusiones tan definitivas, pero los resultados son comparativamente similares a lo observado en ET19 y en Oy50.

Quizás, el punto más interesante de diferenciación entre los sitios trabajados es la presencia de puntas de proyectil únicamente en la cueva Oy7. Tal como se planteó antes, esta situación se relacionaría con las prácticas implementadas en este espacio, diferenciado de los otros por ser un espacio visitado periódicamente y donde se realizaron prácticas diferentes a las planteadas en ET19, Oy50 y Oy31 (Gastaldi et al. 2016; Quesada et al. 2016; Gheco 2017).

En suma, los instrumentos líticos recuperados en estos sitios muestran el fuerte compromiso que generaron las poblaciones con el cuarzo como materia prima, con los conocimientos sobre la mejor manera de tallarlos, reducirlos y formatizarlos. Las prácticas de reproducción social campesina fueron materializadas con estas herramientas, tendientes a cortar, raspar o perforar distintos objetos. Pero, también, se vincularon a la forma en que se construyen los paisajes sociales, las relaciones con otros seres y el establecimiento de territorios campesinos en esta escala local.

Para terminar, nos interesa hacer una reflexión acerca de las posibilidades interpretativas que otorgan estos materiales y evaluar su potencialidad, más allá de la clara simpleza en la preparación de estos instrumentos. Creemos que es imprescindible tener en cuenta que, más allá de estos dos aspectos, existe un cúmulo de conocimientos técnicos, prácticos y espaciales que se articulan para comprender cómo se manufacturaron y utilizaron los instrumentos líticos de cuarzo en la Sierra de El Alto-Ancasti durante el primer milenio de la era.

Hemos podido observar que los talladores y las talladoras conocían como obtener ciertas formas base de acuerdo a las necesidades funcionales que tuviesen, teniendo en cuenta también las restricciones mecánicas del cuarzo. Estos conocimientos debieron incluir la observación y posiblemente el testeo de nódulos para evaluar su potencialidad para la extracción de formas base. Entonces, estas poblaciones sabían exactamente qué tipo de instrumento era necesario preparar para cada una de las actividades diarias o esporádicas que debían realizar. Además, estos conocimientos debieron cruzarse con el cuidado y mantenimiento de las parcelas agrícolas, con 
la crianza de animales, con las elecciones sobre cuál animal sería carneado, con aquellos lugares donde debían trasladarse para conseguir buena madera para reparar los tirantes de sus casas, entre otras actividades. Todo esto, entonces, se vincularía con un conocimiento específico de la talla del cuarzo y la preparación de instrumentos para la realización de múltiples actividades vinculadas a las particularidades de ocupación de los sitios estudiados, que se vinculaba a un cúmulo de saberes locales en torno a múltiples prácticas sociales.

\section{AGRADECIMIENTOS}

Las investigaciones fueron realizadas gracias a subsidios otorgadas por la Universidad Nacional de Catamarca, Agencia Nacional de Promoción Científica y Tecnológica, CONICET y la Secretaría de Ciencia y Tecnología de la Provincia de Catamarca. Queremos agradecer a Gisela Sario por aportarnos consejos y bibliografía importante. Además, a todos los miembros del Equipo Interdisciplinario de El Alto-Ancasti por el apoyo en la realización de las tareas de campo y de laboratorio. Nuestro agradecimiento a los evaluadores por sus comentarios que permitieron el enriquecimiento de este trabajo.

\section{NOTAS}

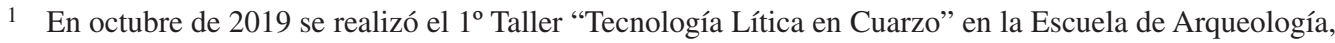
UNCA, en el cual se discutieron algunos de los criterios tecnológicos que aquí se utilizan.

2 Para más información sobre los datos generales de los contextos manufacturados en cuarzo en la Sierra de El Alto-Ancasti ver Moreno y Sentinelli 2014; Moreno 2015; Egea 2016, 2018; Moreno y Egea 2016, 2020.

3 Se denomina compuestos a aquellos instrumentos en donde dos o más grupos tipológicos tienen lugar en una misma unidad artefactual, mientras que no compuestos son aquellos que presentan dos o más filos de igual grupo tipológico (Aschero 1975, 1983).

\section{BIBLIOGRAFÍA}

Aceñolaza, F., H. Miller H. y A. Toselli

1983. Las rocas cristalinas de la Sierra de Ancasti en el contexto de las Sierras Pampeanas Septentrionales.

En F. Aceñolaza, H. Miller y A. Toselli (eds.), Geología de las Sierras de Ancasti: 13-22. Munich. Munstersche Forschungen zur Geologie und Palaontologie 59.

Ahumada, M., D. Egea y E. Moreno

2019. Historias locales, eventos y materiales en la reproducción de la escala doméstica en el este de Catamarca. Trabajo presentado en el XX Congreso Nacional de Arqueología Argentina. Universidad Nacional de Córdoba, Córdoba, Argentina.

Ahumada, M. y E. Moreno

2015-16. La escala doméstica y los animales. Tratamiento diferencial de partes esqueletarias y distribución diferencial intra-sitio en El Taco 19 (El Alto-Ancasti, Catamarca). Anales de Arqueología y Etnología 70/71: 105-118.

Aschero, C.

1975. Ensayo para una clasificación morfológica de artefactos líticos aplicada a estudios tipológicos comparativos. Informe presentado al Consejo Nacional de Investigaciones Científicas y Técnicas. Ms. 1983. Ensayo para una clasificación morfológica de artefactos líticos. Apéndice A y B. Cátedra de Ergología y Tecnología. Facultad de Filosofía y Letras. Universidad de Buenos Aires. Ms. 
Aschero, C. A. y S. Hocsman

2004. Revisando cuestiones tipológicas en torno a la clasificación de artefactos bifaciales. En M. Ramos, A. Acosta y D. Loponte (comps.), Temas de arqueología. Análisis lítico: 7-25. Luján, Universidad Nacional de Luján.

Ballin, T. B.

2004. The worked quartz vein at Cnoc Dubh, Isle of Lewis, Western Isles. Presentation and discussion of a small prehistoric quarry, Scottish Archaeological Internet Reports (SAIR) 11. http://www.sair. org.uk/ sair11/index.html

2008. Quartz Technology in Scottish Prehistory. Scottish Archaeological Internet Reports (SAIR) 26. http://www.sair.org.uk/sair26/index.html.

Barot, C.

2017. Las vasijas en la vida diaria. Análisis morfológico-funcional del material cerámico de una casa emplazada en las Sierras de El Alto-Ancasti (siglos VII y VIII d.C.). Tesis de Licenciatura inédita. Escuela de Arqueología. Universidad Nacional de Catamarca.

Barot, C., M. Gastaldi, M. V. Zucchi, F. Marte, N. Mastrangelo, R. Álvarez, S. Pereda y L. Gheco 2019. Las vasijas pintadas de El Taco 19: transformaciones y continuidades en los modos de producción de la cerámica Aguada desde una perspectiva arqueométrica (frx, sem-eds y raman) e histórica. Trabajo presentado en el XX Congreso Nacional de Arqueología Argentina. Universidad Nacional de Córdoba, Córdoba.

Beardsell, R.

2013. Mass and atribute analysis of the quartz lithic assemblage from the Grandfather Quarry (HbMd4) near Granville Lake, Northern Manitoba. Tesis doctoral inédita. Departamento de Arqueología, Universidad de Manitoba, Winnipeg, Canadá.

Bisson, M. S.

1990. Lithic reduction sequences as an aid to the analysis of Late Stone Age quartz assemblages from the Luano Spring, Chingola, Zambia. The African Archaeological Review 8: 103-138.

Borgo, M., G. Heider, A. Ortiz Suarez, C. Gardini y R. Curtoni

2019. Primeros resultados de los estudios líticos en el sitio Quebrada del Chiquero (provincia de San Luis, Argentina). Anales de Arqueología y Etnología 74(1): 39-56.

Brizuela, C.

2018. Entre gubias, escoplos y cinceles: Una caracterización funcional de base microscópica, para instrumentos líticos experimentales. Tesis de Licenciatura inédita. Facultad de Filosofía y Humanidades. Universidad Nacional de Córdoba.

Byrne, F., T. Proffitt, A. Arroyo e I. de la Torre

2016. A comparative analysis of bipolar and freehand experimental knapping products from Olduvai Gorge, Tanzania. Quaternary International 424: 58-68. http://dx.doi.org/10.1016/j. quaint.2015.08.018

Callahan, E.

1987. An Evaluation of the Lithic Technology in Middle Sweden During the Mesolithic and Neolithic. Uppsala: Societas Archaeologica Upsaliensis.

Caminoa, J.

2016. Un estudio de tecnología lítica desde la antropología de las técnicas: El caso del Alero Deodoro Roca ca. 3000 AP, Ongamira, Ischilín, Córdoba. Oxford. Archaeopress Publishing. 
Carbonelli, J.

2011. "Motivos porque y para" en la tecnología lítica de un sitio formativo en el valle de Yokavil, Provincia de Catamarca. Intersecciones en Antropología 12: 31-44.

Carrera Aizpitarte, M.

2017. Avances en los estudios arqueológicos desarrollados en las Sierras de San Luis. El sitio club El Trapiche (departamento Coronel Pringles, provincia de San Luis). Anales de Arqueología y Etnología 72(2): 191-217.

De la Peña, P.

2011. Sobre la identificación macroscópica de las piezas astilladas: propuesta experimental. Trabajos de Prehistoria 68(1): 79-98.

De la Peña, P. y L. Wadley

2014. Quartz Knapping Strategies in the Howiesons Poort at Sibudu (KwaZulu-Natal, South Africa). PLOS ONE 9(7): e101534. doi:10.1371/journal.pone.0101534

De Lombera Hermida, A.

2009. The scar identification of lithic quartz industries. En: F. Sternke, L. Eigeland y L. Costa (eds.), Non-Flint Raw Material Use in Prehistory Old Prejudices and New Directions: 5-11. Oxford. British Archaeological Reports.

Diez Martín, F. P., Sánchez Yustos, M. Domínguez-Rodrigo, A. Mabulla y R. Barba

2009. Where Olduvai hominins making butchering tools or battering tools? Analysis of a recently excavated lithic assemblage from BK (Bed II, Olduvai Gorge, Tanzania). Journal of Anthropological Archaeology 28: 274-289.

Dlugosz, J. C.

2005. Prospecciones arqueológicas en los sitios Los Pedraza y Los Corpitos, dpto. El Alto, pcia. de Catamarca. Tesis de licenciatura inédita, Facultad de Ciencias Naturales e Instituto Miguel Lillo, Universidad Nacional de Tucumán, Tucumán.

Driscoll, K.

2009. Exploring the Chaine opératoires in Irish quartz lithic traditions: current research. Internet Archaeology 26. http://intarch.ac.uk/journal/issue26/driscollbindex.html

2010. Understanding Quartz Technology in Early Prehistoric Ireland. Tesis Doctoral inédita, UCD School of Archaeology, University College Dublin, Dublin.

2011. Vein quartz in lithic traditions: an analysis based on experimental archaeology. Journal of Archaeological Science 38: 734-745.

Egea, D.

2016. Prácticas tecnológicas líticas entre las sociedades del este catamarqueño durante la segunda mitad del Primer milenio D.C. La Zaranda de Ideas 14 (1): 55-70.

2018. Tecnología lítica en la Sierra de El Alto-Ancasti (Catamarca). Aporte desde la experimentación. Revista del Museo de Antropología 11(2): 39-48.

Egea, D. e I. Gerola

2020. El uso del cuarzo en la Sierra El Alto-Ancasti (Catamarca, Argentina). Experimentación y casos arqueológicos. Revista del Museo de Antropología, 13(1): 155-160.https://doi.org/10.31048/1852.4826. v13.n1.23827

Egea, D., S. López y J. Quiroga Viñas

2019. Un abordaje multidimensional del sitio Oyola 50. Aproximaciones desde la estratigrafía y el análisis lítico y cerámico a la historia de una vivienda en el este catamarqueño. Trabajo presentado en el $X X$ Congreso Nacional de Arqueología Argentina. Córdoba, Argentina. 
D. EGEA Y E. MoRENo - INSTRUMENTOS LítICOS DE CUARZO, PRÁCTICAS SOCIALES Y VIDA CAMPESINA DURANTE ...

Escola, P.

2000. Tecnología lítica y sociedades agropastoriles tempranas. Tesis Doctoral inédita. Facultad de Filosofía y Letras. Universidad Nacional de Buenos Aires. Buenos Aires.

Fábregas Valcarce, R. y C. Rodríguez Rellán

2008. Gestión del cuarzo y la pizarra en el Calcolítico Peninsular: El "Santuario" de El Pedroso (Trabazos de Aliste, Zamora). Trabajos de Prehistoria 65 (1): 125-142.

Franco Salvi, V., J. Salazar y J. Montegú

2016. Prácticas cotidianas y vida aldeana. Un análisis desde la tecnología lítica en el Valle de Tafí(Tucumán, Argentina). Cuadernos del Instituto de Antropología y Pensamiento Latinoamericano 25(2): 141-158.

Gaál, E.

2014. Decisiones tecnológicas y producción lítica en el sur del Valle de Yocavil (pcia. de Catamarca). Un estudio comparativo de conjuntos artefactuales tempranos y tardíos. Tesis de Licenciatura inédita. Facultad de Filosofía y Letras, Universidad de Buenos Aires.

Gastaldi, M., L. Gheco, E. Moreno, G. Granizo, M. Ahumada, D. Egea y M. Quesada

2016. Primeros resultados de las excavaciones estratigráficas en Oyola 7 (Sierra de El Alto-Ancasti, provincia de Catamarca, Argentina). Comechingonia 20: 73-104.

Gerola, I.

2018. El hábito de tallar el paisaje verde. Tecnología lítica en el sector septentrional de El Alto - Ancasti (Catamarca). Tesis de Licenciatura inédita. Facultad de Filosofía y Letras, Universidad de Buenos Aires.

Gheco, L.

2012. Una historia en la pared. Hacia una visión diacrónica del arte rupestre de Oyola. Tesis de Licenciatura inédita, Escuela de Arqueología, Universidad Nacional de Catamarca, Catamarca.

2017. El laberinto de las paredes pintadas. Una historia de los abrigos con arte rupestre de Oyola, Catamarca. Tesis doctoral inédita. Facultad de Filosofía y Humanidades, Universidad Nacional de Córdoba.

Gordillo, I., L. Eguía y V. Zuccarelli

2017. Las casas del sol naciente. Arqueología de la Vertiente Oriental del Alto-Ancasti. En B. Ventura, G. Ortizy M. Cremonte (eds.), Arqueología de la Vertiente oriental Surandina. Interacción macro-regional, materialidades, economía y ritualidad: 111-134. Buenos Aires. Sociedad Argentina de Antropología.

Hogg, A., T. Heaton, Q. Hua, J. Palmer, C. Turney, J. Southon, A. Bayliss, P. Blackwell, G. Boswijk, C. Bronk Ramsey, C. Pearson, F. Petchey, P. Reimer, R. Reimer y L. Wacker

2020. SHCal20 Southern Hemisphere Calibration, 0-55,000 years cal BP. Radiocarbon 62(4): 759-778.

Knutsson, K.

1998. Convention and lithic analysis. En L Holm y K. Knutsson (eds.), Third Flint Alternatives Conference at Uppsala. OPIA 16: 71-93. Uppsala. Department of Archaeology and Ancient History, Uppsala University.

Manninen, M. A.

2016. The effect of raw material properties on flake and flake-tool dimensions: A comparison between quartz and chert. Quaternary International, 424: 24-31.

Mogni, V., L. Oakley, H. Maturo, L. Galleti y D. Eros Prado

2015. Biogeografía y florística de los bosques secos estacionales neotropicales (BSEN). Revista OKARA, Geografía en debate, 9(2): 275-296.

Montegú, J.

2018. Rocas, tecnología y vida aldeana durante el Primer Milenio de la Era en Anfama (Dto. Tafí Viejo, 
Tucumán, Rep. Argentina). Tesis de Licenciatura inédita. Facultad de Filosofía y Humanidades. Escuela de Historia. Universidad Nacional de Córdoba.

Moreno, E.

2015. Materias primas, instrumentos líticos y prácticas domésticas en las serranías de El Alto-Ancasti, Catamarca. Cuadernos INAPL-Series Especiales 2 (2): 141-160.

Moreno, E. y M. Ahumada

2018. Animales y humanos en las cumbres de Ancasti (siglos VIII y IX d.C.). Paisajes campesinos y recursos locales. Archaeofauna. International Journal of Archaeozoology 27: 195-208.

Moreno, E. y D. Egea

2016. Visitas en el tiempo. Tecnología lítica de una cueva con arte rupestre en el Este catamarqueño. Arqueología 22(1): 223-232.

2020. Técnicas de observación, talla experimental y morfometría geométrica para el estudio de material lítico tallado en cuarzo. Revista del Museo de Antropología 13(1): 301-306. https://doi. org/10.31048/1852.4826.v13.n1.23836

Moreno, E., G. Sario, E. Gaál, D. Egea, I. Gerola, C. Brizuela y J. Montegú.

2021. Aportes metodológicos para el estudio de la tecnología lítica tallada en cuarzo. Arqueología en evaluación.

Moreno, E., C. Samec y M. Ahumada

2019. Producción pastoril en el este catamarqueño. Una aproximación desde los análisis de isótopos estables. Trabajo presentado en el IV Congreso Nacional de Zooarqueología Argentina. Escuela de Arqueología, Universidad Nacional de Catamarca.

Moreno, E. y N. Sentinelli

2014. Tecnología lítica en las sierra de El Alto-Ancasti, Catamarca. Cuadernos №45: 95-105.

Mórlans, C.

1995. Regiones Naturales de Catamarca. Provincias Geológicas y Fitogeográficas. Revista de Ciencia y Técnica. 2 (2): 1-36.

Mourre, V.

1996. Les industries en quartz au Paléolithique. Terminologie, Methodologie et Technologie. Paleo 8: 205-223.

Nazar, D.

2003. Relevamiento arqueológico de la zona austral de la sierra de Ancasti (Provincia de Catamarca). CENEDIT, Universidad Nacional de Catamarca.

Orton, J.

2008. A useful measure of the desirability of different raw materials for retouch within and between assemblages: the raw material retouch index (RMRI). Journal of Archaeological Science 35, 10901094. doi:10.1016/j.jas.2007.08.002

Pargeter, J. y P. De la Peña

2017. Milky quartz bipolar reduction and lithic miniaturization: Experimental results and archaeological implications. Journal of Field Archaeology 42(6): 551-565.

Pautassi, E.

2018. Quebrando rocas. Una aproximación metodológica para el estudio del cuarzo en contextos arqueológicos de Córdoba (Argentina). Archaeopress Publishing LTD. Oxford. 
D. EgEA y E. Moreno - InSTRUMENTOS Líticos de CUARZo, PRÁCtICAS SOCIALES Y VIDA CAMPESINA DURANTE ...

Pautassi, E. y G. Sario

2014. La talla de reducción: aproximaciones experimentales para el estudio del cuarzo.Arqueoweb, 15:3-17.

Pazzarelli, F.

2011. Arqueología de la comida. Cultura material y prácticas de alimentación en Ambato, Catamarca, (Argentina). Siglos V-XI d.C. Tesis doctoral inédita. Facultad de Filosofía y Humanidades. Universidad Nacional de Córdoba.

2013. Otros-maíces. Trayectorias y transformaciones culinarias del maíz en Ambato (Catamarca, Noroeste Argentino). Revista Española de Antropología Americana 43(2): 329-351.

Prous, A. P.

2004. Apuntes para análisis de industrias líticas. Ortegalia. Monografías de Arqueoloxía, Historia e Patrimonio 2. Ortigueira: Fundación Federico Maciñeira.

Quesada, M. y M. Gastaldi

2013. Devenir Casa. Trabajo presentado en el VII Congreso de la Asociación de Estudios Bolivianos. Sucre. Bolivia.

Quesada, M., M. Gastaldi y G. Granizo

2012. Construcción de periferias y producción de lo local en las cumbres de El Alto-Ancasti. Relaciones de la Sociedad Argentina de Antropología XXXVII (2): 435-456.

Quesada, M., V. Zuccarelli, L. Gheco, M. Gastaldi, S. Boscatto y E. Moreno

2016. Paisaje y experiencia en Oyola a finales del Primer Milenio d.C. (dpto. El Alto, Catamarca). Comechingonia 20: 13-42.

Quiroga Viñas, J.

2020. Los espacios residenciales y la vida cotidiana en El Alto-Ancasti. El caso del sitio Oyola 50. Tesis de licenciatura inédita. Facultad de filosofía y humanidades, Universidad Nacional de Córdoba.

Reinoso, D.

2017. Tecnología lítica del sitio Barranca I (Córdoba, Argentina): avances en el registro de las fuentes inmediatas de cuarzo. Revista Sociedades de Paisajes Áridos y Semi-Áridos X: 195-220.

Sánchez Yustos, P., J. García Garriga y K. Martínez

2017. Experimental approach to the study of the European Mode 1 lithic record: The bipolar core technology at Vallparadis (Barcelona, Spain). European Journal of Archeology 20(2): 1-24.

Sario, G.

2008. Tecnología bifacial en las Sierras de San Luis y depresión del Conlara (provincia de San Luis, República Argentina) en el Holoceno temprano. Arqueoweb. Revista sobre arqueología en Internet, 10 (1): 1-18.

Sario, G. y E. Pautassi

2015. Canteras-taller de cuarzo y un análisis de los conjuntos artefactuales del sitio Piedra Blanca (Copacabana, Córdoba). Revista Arqueología 21 (2): 165-175.

Sario, G. y M. Salvatore

2018. Caracterización petrográfica y disponibilidad de recursos líticos en la cuenca del río Copacabana, noroeste de Córdoba, Argentina. Mundo de Antes 12: 43-66.

Sentinelli, N.

2012. Tecnología lítica en una "cocina" del valle del Cajón (Dto. de Santa María). Una perspectiva microescalar. Tesis de Licenciatura inédita. Escuela de Arqueología, Universidad Nacional de Catamarca. 
Relaciones de la Sociedad Argentina de Antropología 46 (1), enero-junio 2021: 145-176

Tallavaara, M., M. Manninen, E. Hertell y T. Rankama

2010. How flakes shatter. A critical evaluation of fracture analysis. Journal of Archaeological Science 37(10): 2442-2448.

Vilches, J.

2016. Análisis lítico del poblado arqueológico Rincón del Toro en un contexto Aguada tardío: Tendencias tecnológicas en un ambiente de hostilidad regional desde fines del período medio y comienzos del intermedio tardío ( $c a .750-950$ a 1200-1400 d.C.) en el Valle de Vinchina, oeste de la Pcia. de La Rioja, Argentina. Tesis de Licenciatura inédita. Facultad de Filosofía y Letras. Universidad de Buenos Aires.

\section{Zuccarelli, V.}

2012. Paisajes de producción y reproducción durante el Periodo de Integración Regional: uso de GIS para el análisis de la agricultura en El Alto-Ancasti. Tesis de Licenciatura inétida, Facultad de Filosofía y Letras, Universidad de Buenos Aires.

2020. Desde las cumbres a las yungas: Las múltiples escalas de las prácticas agrarias prehispánicas en las Sierra de El Alto-Ancasti (Catamarca) durante el primer milenio AD. Tesis doctoral inédita. Facultad de Filosofía y Letras, Universidad de Buenos Aires. 